\title{
THE SPECTRUM OF ISOTROPIC DIFFUSE GAMMA-RAY EMISSION BETWEEN 100 MeV AND 820 GeV
}

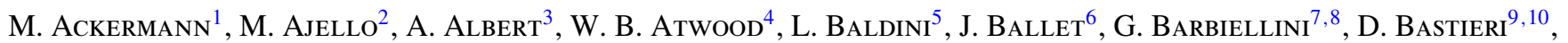

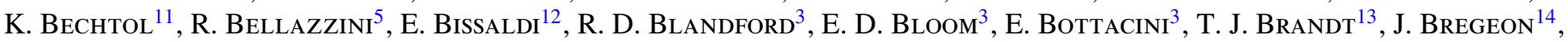
P. Bruel ${ }^{15}$, R. Buehler ${ }^{1}$, S. Buson ${ }^{9}, 10$, G. A. Caliandro ${ }^{3,16}$, R. A. Cameron ${ }^{3}$, M. Caragiulo ${ }^{17}$, P. A. Caraveo ${ }^{18}$, E. Cavazzuti ${ }^{19}$, C. Cecchi $^{20,21}$, E. Charles ${ }^{3}$, A. Chekhtman ${ }^{22,67}$, J. Chiang ${ }^{3}$, G. Chiaro ${ }^{10}$, S. Ciprini ${ }^{19,23}$, R. Claus ${ }^{3}$, J. Cohen-Tanugi ${ }^{14}$, J. Conrad ${ }^{24,25,26,68}$, A. Cuoco ${ }^{25,27,28}$, S. Cutini ${ }^{19,23}$, F. D' Ammando ${ }^{29,30}$, A. De Angelis ${ }^{31}$,

F. De Palma ${ }^{17,32}$, C. D. Dermer ${ }^{33}$, S. W. Digel ${ }^{3}$, E. do Couto e Silva ${ }^{3}$, P. S. Drell ${ }^{3}$, C. Favuzzi ${ }^{17,34}$, E. C. Ferrara ${ }^{13}$,

W. B. Focke ${ }^{3}$, A. Franckowiak ${ }^{3}$, Y. FukazaWA ${ }^{35}$, S. FunK ${ }^{3}$, P. Fusco ${ }^{17,34}$, F. Gargano ${ }^{17}$, D. Gasparrini ${ }^{19,23}$, S. Germani ${ }^{20,21}$, N. Giglietto ${ }^{17,34}$, P. Giommi ${ }^{19}$, F. Giordano ${ }^{17,34}$, M. Girolettio ${ }^{29}$, G. Godfrey ${ }^{3}$, G. A. GomeZ-Vargas ${ }^{36,37}$,

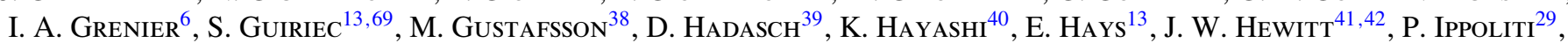
T. Jogler ${ }^{3}$, G. JóhANNESSON ${ }^{43}$, A. S. JOHNSON ${ }^{3}$, W. N. JOHNSON ${ }^{33}$, T. KAMAE ${ }^{3}$, J. KATAOKA ${ }^{44}$, J. KNÖDLSEDER ${ }^{45,46}$, M. Kuss ${ }^{5}$,

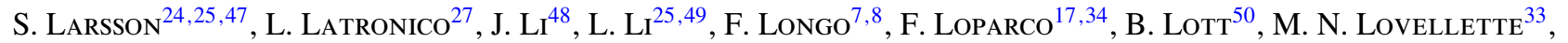

P. Lubrano ${ }^{20,21}$, G. M. MAdejski ${ }^{3}$, A. MANFreda ${ }^{5}$, F. Massaro ${ }^{51}$, M. MAYer ${ }^{1}$, M. N. MAZziotta ${ }^{17}$, J. E. McEneri ${ }^{13,52}$, P. F. Michelson ${ }^{3}$, W. Mitthumsiri ${ }^{53}$, T. Mizuno ${ }^{54}$, A. A. Moiseev $^{42,52}$, M. E. MonZani ${ }^{3}$, A. Morselli ${ }^{36}$, I. V. Moskalenko ${ }^{3}$, S. Murgia ${ }^{55}$, R. Nemmen ${ }^{13,41,42}$, E. Nuss ${ }^{14}$, T. Ohsugi ${ }^{54}$, N. Omodei ${ }^{3}$, E. Orlando ${ }^{3}$, J. F. Ormes ${ }^{56}$, D. Paneque ${ }^{3,57}$,

J. H. Panetta ${ }^{3}$, J. S. Perkins ${ }^{13}$, M. Pesce-Rollins ${ }^{5}$, F. Piron ${ }^{14}$, G. Pivato ${ }^{5}$, T. A. Porter ${ }^{3}$, S. Rainò ${ }^{17,34}$, R. Rando ${ }^{9,10}$, M. Razzano ${ }^{5,70}$, S. Razzaque ${ }^{58}$, A. Reimer ${ }^{3,39}$, O. Reimer ${ }^{3,39}$, T. Reposeur ${ }^{50}$, S. Ritz ${ }^{4}$, R. W. Romani ${ }^{3}$, M. SÁnChez-Conde ${ }^{3}$, M. SchaAl ${ }^{59,67}$, A. Schulz ${ }^{1}$, C. Sgrò ${ }^{5}$, E. J. Siskind ${ }^{60}$, G. SPANDRe ${ }^{5}$, P. Spinelli ${ }^{17,34}$, A. W. Strong ${ }^{61}$, D. J. Suson ${ }^{62}$,

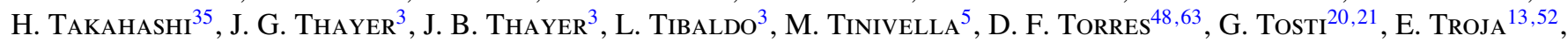

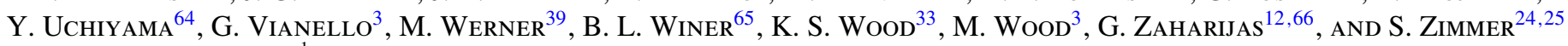

${ }^{1}$ Deutsches Elektronen Synchrotron DESY, D-15738 Zeuthen, Germany; markus.ackermann@ desy.de

${ }^{2}$ Department of Physics and Astronomy, Clemson University, Kinard Lab of Physics, Clemson, SC 29634-0978, USA

${ }^{3}$ W. W. Hansen Experimental Physics Laboratory, Kavli Institute for Particle Astrophysics and Cosmology, Department of Physics and SLAC National Accelerator Laboratory, Stanford University, Stanford, CA 94305, USA

${ }^{4}$ Santa Cruz Institute for Particle Physics, Department of Physics and Department of Astronomy and Astrophysics, University of California at Santa Cruz, Santa Cruz, CA 95064, USA

${ }^{5}$ Istituto Nazionale di Fisica Nucleare, Sezione di Pisa, I-56127 Pisa, Italy

${ }^{6}$ Laboratoire AIM, CEA-IRFU/CNRS/Université Paris Diderot, Service d'Astrophysique, CEA Saclay, F-91191 Gif sur Yvette, France

${ }^{7}$ Istituto Nazionale di Fisica Nucleare, Sezione di Trieste, I-34127 Trieste, Italy

${ }^{8}$ Dipartimento di Fisica, Università di Trieste, I-34127 Trieste, Italy

${ }^{9}$ Istituto Nazionale di Fisica Nucleare, Sezione di Padova, I-35131 Padova, Italy

${ }^{10}$ Dipartimento di Fisica e Astronomia “G. Galilei,” Università di Padova, I-35131 Padova, Italy

${ }^{11}$ Kavli Institute for Cosmological Physics, University of Chicago, Chicago, IL 60637, USA; bechtol@kicp.uchicago.edu

12 Istituto Nazionale di Fisica Nucleare, Sezione di Trieste, and Università di Trieste, I-34127 Trieste, Italy

${ }_{13}$ NASA Goddard Space Flight Center, Greenbelt, MD 20771, USA

${ }^{14}$ Laboratoire Univers et Particules de Montpellier, Université Montpellier 2, CNRS/IN2P3, F-34095 Montpellier, France

${ }^{15}$ Laboratoire Leprince-Ringuet, École Polytechnique, CNRS/IN2P3, F-91128 Palaiseau, France

${ }^{16}$ Consorzio Interuniversitario per la Fisica Spaziale (CIFS), I-10133 Torino, Italy

${ }^{17}$ Istituto Nazionale di Fisica Nucleare, Sezione di Bari, I-70126 Bari, Italy

${ }^{18}$ INAF-Istituto di Astrofisica Spaziale e Fisica Cosmica, I-20133 Milano, Italy

${ }^{19}$ Agenzia Spaziale Italiana (ASI) Science Data Center, I-00133 Roma, Italy

${ }^{20}$ Istituto Nazionale di Fisica Nucleare, Sezione di Perugia, I-06123 Perugia, Italy

${ }^{21}$ Dipartimento di Fisica, Università degli Studi di Perugia, I-06123 Perugia, Italy

${ }^{22}$ Center for Earth Observing and Space Research, College of Science, George Mason University, Fairfax, VA 22030, USA

${ }^{23}$ INAF Osservatorio Astronomico di Roma, I-00040 Monte Porzio Catone (Roma), Italy

${ }^{24}$ Department of Physics, Stockholm University, AlbaNova, SE-106 91 Stockholm, Sweden

25 The Oskar Klein Centre for Cosmoparticle Physics, AlbaNova, SE-106 91 Stockholm, Sweden

${ }^{26}$ The Royal Swedish Academy of Sciences, Box 50005, SE-104 05 Stockholm, Sweden

${ }^{27}$ Istituto Nazionale di Fisica Nucleare, Sezione di Torino, I-10125 Torino, Italy

${ }^{28}$ Dipartimento di Fisica Generale “Amadeo Avogadro," Università degli Studi di Torino, I-10125 Torino, Italy

${ }^{29}$ INAF Istituto di Radioastronomia, I-40129 Bologna, Italy

${ }^{30}$ Dipartimento di Astronomia, Università di Bologna, I-40127 Bologna, Italy

${ }^{31}$ Dipartimento di Fisica, Università di Udine and Istituto Nazionale di Fisica Nucleare, Sezione di Trieste, Gruppo Collegato di Udine, I-33100 Udine, Italy

${ }^{32}$ Università Telematica Pegaso, Piazza Trieste e Trento 48, I-80132 Napoli, Italy

${ }^{33}$ Space Science Division, Naval Research Laboratory, Washington, DC 20375-5352, USA

${ }^{34}$ Dipartimento di Fisica "M. Merlin" dell'Università e del Politecnico di Bari, I-70126 Bari, Italy

${ }^{35}$ Department of Physical Sciences, Hiroshima University, Higashi-Hiroshima, Hiroshima 739-8526, Japan

${ }^{36}$ Istituto Nazionale di Fisica Nucleare, Sezione di Roma “Tor Vergata," I-00133 Roma, Italy

${ }^{37}$ Departamento de Fisíca, Pontificia Universidad Católica de Chile, Avenida Vicuña Mackenna 4860, Santiago, Chile

${ }^{38}$ Service de Physique Theorique, Universite Libre de Bruxelles (ULB), Bld du Triomphe, CP225, B-1050 Brussels, Belgium

${ }^{39}$ Institut für Astro- und Teilchenphysik and Institut für Theoretische Physik, Leopold-Franzens-Universität Innsbruck, A-6020 Innsbruck, Austria

${ }^{40}$ Institute of Space and Astronautical Science, Japan Aerospace Exploration Agency, 3-1-1 Yoshinodai, Chuo-ku, Sagamihara, Kanagawa 252-5210, Japan

${ }^{41}$ Department of Physics and Center for Space Sciences and Technology, University of Maryland Baltimore County, Baltimore, MD 21250, USA

${ }^{42}$ Center for Research and Exploration in Space Science and Technology (CRESST) and NASA Goddard Space Flight Center, Greenbelt, MD 20771, USA

${ }^{43}$ Science Institute, University of Iceland, IS-107 Reykjavik, Iceland

${ }^{44}$ Research Institute for Science and Engineering, Waseda University, 3-4-1, Okubo, Shinjuku, Tokyo 169-8555, Japan

${ }^{45}$ CNRS, IRAP, F-31028 Toulouse cedex 4, France 


\author{
${ }^{46}$ GAHEC, Université de Toulouse, UPS-OMP, IRAP, F-31400 Toulouse, France \\ ${ }^{47}$ Department of Astronomy, Stockholm University, SE-106 91 Stockholm, Sweden \\ ${ }^{48}$ Institute of Space Sciences (IEEC-CSIC), Campus UAB, E-08193 Barcelona, Spain \\ 49 Department of Physics, KTH Royal Institute of Technology, AlbaNova, SE-106 91 Stockholm, Sweden \\ ${ }^{50}$ Centre d'Études Nucléaires de Bordeaux Gradignan, IN2P3/CNRS, Université Bordeaux 1, BP120, F-33175 Gradignan Cedex, France \\ ${ }^{51}$ Department of Astronomy, Department of Physics and Yale Center for Astronomy and Astrophysics, Yale University, New Haven, CT 06520-8120, USA \\ 52 Department of Physics and Department of Astronomy, University of Maryland, College Park, MD 20742, USA \\ ${ }^{53}$ Department of Physics, Faculty of Science, Mahidol University, Bangkok 10400, Thailand \\ ${ }^{54}$ Hiroshima Astrophysical Science Center, Hiroshima University, Higashi-Hiroshima, Hiroshima 739-8526, Japan \\ ${ }^{55}$ Center for Cosmology, Physics and Astronomy Department, University of California, Irvine, CA 92697-2575, USA \\ ${ }^{56}$ Department of Physics and Astronomy, University of Denver, Denver, CO 80208, USA \\ ${ }^{57}$ Max-Planck-Institut für Physik, D-80805 München, Germany \\ ${ }^{58}$ Department of Physics, University of Johannesburg, P.O. Box 524, Auckland Park 2006, South Africa \\ ${ }^{59}$ National Research Council Research Associate, National Academy of Sciences, Washington, DC 20001, USA \\ ${ }^{60}$ NYCB Real-Time Computing Inc., Lattingtown, NY 11560-1025, USA \\ ${ }^{61}$ Max-Planck Institut für Extraterrestrische Physik, D-85748 Garching, Germany \\ 62 Department of Chemistry and Physics, Purdue University Calumet, Hammond, IN 46323-2094, USA \\ ${ }^{63}$ Institució Catalana de Recerca i Estudis Avançats (ICREA), E-08028 Barcelona, Spain \\ ${ }^{64}$ 3-34-1 Nishi-Ikebukuro, Toshima-ku, Tokyo 171-8501, Japan \\ ${ }^{65}$ Department of Physics, Center for Cosmology and Astro-Particle Physics, The Ohio State University, Columbus, OH 43210, USA \\ ${ }^{66}$ The Abdus Salam International Center for Theoretical Physics, Strada Costiera 11, Trieste I-34151, Italy \\ Received 2014 June 19; accepted 2014 September 29; published 2015 January 19
}

\begin{abstract}
The $\gamma$-ray sky can be decomposed into individually detected sources, diffuse emission attributed to the interactions of Galactic cosmic rays with gas and radiation fields, and a residual all-sky emission component commonly called the isotropic diffuse $\gamma$-ray background (IGRB). The IGRB comprises all extragalactic emissions too faint or too diffuse to be resolved in a given survey, as well as any residual Galactic foregrounds that are approximately isotropic. The first IGRB measurement with the Large Area Telescope (LAT) on board the Fermi Gamma-ray Space Telescope (Fermi) used 10 months of sky-survey data and considered an energy range between $200 \mathrm{MeV}$ and $100 \mathrm{GeV}$. Improvements in event selection and characterization of cosmic-ray backgrounds, better understanding of the diffuse Galactic emission (DGE), and a longer data accumulation of 50 months allow for a refinement and extension of the IGRB measurement with the LAT, now covering the energy range from $100 \mathrm{MeV}$ to $820 \mathrm{GeV}$. The IGRB spectrum shows a significant high-energy cutoff feature and can be well described over nearly four decades in energy by a power law with exponential cutoff having a spectral index of $2.32 \pm 0.02$ and a break energy of $(279 \pm 52) \mathrm{GeV}$ using our baseline DGE model. The total intensity attributed to the IGRB is $(7.2 \pm 0.6) \times 10^{-6} \mathrm{~cm}^{-2} \mathrm{~s}^{-1} \mathrm{sr}^{-1}$ above $100 \mathrm{MeV}$, with an additional $+15 \% /-30 \%$ systematic uncertainty due to the Galactic diffuse foregrounds.
\end{abstract}

Key words: diffuse radiation - gamma rays: diffuse background

Supporting material: machine-readable table

\section{INTRODUCTION}

The universe is filled with electromagnetic radiation, which can be characterized by a cosmological energy density and spectrum. This extragalactic background light (EBL) is energetically dominated by thermal relic radiation from the last scattering surface observed as the cosmic microwave background. Different physical processes characterize the EBL in each wavebandstarlight in the optical, thermal dust emission in the infrared, and emission from active galactic nuclei (AGNs) in X-rays. The extragalactic $\gamma$-ray background (EGB) provides a nonthermal perspective on the cosmos, which is also explored through the cosmic radio background, as well as extragalactic cosmic rays (CRs) and neutrinos.

The EGB represents a superposition of all $\gamma$-ray sources, both individual and diffuse, from the edge of the Milky Way to the edge of the observable universe, and is thus expected to encode diverse phenomena (see Dermer 2007 for a comprehensive

\footnotetext{
${ }^{67}$ Resident at Naval Research Laboratory, Washington, DC 20375, USA.

68 Royal Swedish Academy of Sciences Research Fellow, funded by a grant from the K. A. Wallenberg Foundation.

69 NASA Postdoctoral Program Fellow, USA.

${ }^{70}$ Funded by contract FIRB-2012-RBFR12PM1F from the Italian Ministry of Education, University and Research (MIUR).
}

review). Guaranteed contributions arise from established extragalactic $\gamma$-ray source classes including AGNs, star-forming galaxies, and $\gamma$-ray bursts. The beamed emission from blazars is sufficiently bright that statistically large samples of individual sources have now been detected to cosmological distances (Ackermann et al. 2011a). Accordingly, the flux distribution of blazars even below the detection threshold for individual sources can in principle be estimated from a relatively firm empirical basis through an extrapolation of the observed flux distribution (e.g., Abdo et al. 2010c; Ajello et al. 2012, 2014), although a consensus has not yet been reached (e.g., Singal et al. 2012). For other populations, such as star-forming galaxies (Pavlidou \& Fields 2002; Thompson et al. 2007; Fields et al. 2010; Makiya et al. 2011; Stecker \& Venters 2011; Ackermann et al. 2012c) and AGNs with jets oriented obliquely to our line of sight (Inoue 2011; Di Mauro et al. 2014), the cumulative intensity is almost entirely unresolved by current instruments; calculations of the flux distribution incorporating physical models and/or multiwavelength scaling relations must be invoked to estimate their EGB contributions. There are additional theoretically well-motivated extragalactic source classes too faint to have been individually detected thus far, including galaxy clusters and their associated large-scale structure formation shocks (Colafrancesco \& Blasi 1998; Loeb \& Waxman 2000). 
At energies $\gtrsim 100 \mathrm{GeV}$, the interaction length for $\gamma$-rays with photons of the UV/optical/IR EBL becomes much shorter than a Hubble length, thus defining an effective $\gamma$-ray horizon. The electromagnetic cascades initiated by both very high energy $\gamma$-rays (Coppi \& Aharonian 1997) and ultra high energy CRs (Berezinskii \& Smirnov 1975) interacting with the EBL create truly diffuse EGB contributions.

Finally, more exotic processes such as dark matter annihilation/decay may be present, though as yet unrecognized, in the EGB (Bergström et al. 2001; Ullio et al. 2002; Taylor \& Silk 2003).

From an observational standpoint, there are two main challenges in measuring the EGB. One is to model the diffuse Galactic emission (DGE) created by CR interactions with interstellar gas (ISG) and interstellar radiation fields (ISRFs), which is comparable to the EGB intensity at energies $\gtrsim 1 \mathrm{GeV}$ even at the Galactic poles and therefore represents a strong foreground to the EGB measurement. The second challenge is separating cosmic $\gamma$-rays from CR-induced backgrounds at the detector level. For instruments in low Earth orbit, the CR intensity can exceed that of the EGB signal by a factor of up to $\sim 10^{6}$. In addition, there is a sizable flux of secondary particles that are produced by interactions of CRs in Earth's atmosphere.

The existence of all-sky $\gamma$-ray emission was first realized experimentally using 621 candidate $\gamma$-rays collected by the OSO-3 satellite (Clark et al. 1968; Kraushaar et al. 1972), while Fichtel et al. $(1975,1978)$ reported the first spectral measurement of an isotropic diffuse background using the $S A S-2$ satellite. Analyses using more sensitive instruments capable of detecting individual extragalactic sources began reporting the residual all-sky average intensity after subtracting individual sources and DGE templates (e.g., Sreekumar et al. 1998; Strong et al. 2004, using EGRET). The remaining emission component is found to be approximately isotropic on large angular scales and is commonly called the isotropic diffuse $\gamma$-ray background (IGRB). The sum of the IGRB and individually resolved extragalactic sources represents an upper limit to the total EGB intensity, since residual unresolved Galactic emissions may be present in the IGRB. For example, CR interactions with gas (Feldmann et al. 2013) or radiation fields (Keshet et al. 2004) in the extended halo of the Milky Way, unresolved Galactic sources such as millisecond pulsars (Faucher-Giguère \& Loeb 2010), and CR interactions with solar system debris (Moskalenko \& Porter 2009) and the solar radiation field (Moskalenko et al. 2006; Orlando \& Strong 2007, 2008) have been considered as sources of approximately isotropic emission on large angular scales.

The intensity attributed to the IGRB is observation dependent because more sensitive instruments with deeper exposures can extract fainter extragalactic sources, whereas the total EGB intensity (assuming complete subtraction of all Galactic emissions) is the fundamental quantity.

The Large Area Telescope (LAT) on board the Fermi Gammaray Space Telescope (Fermi) is the first instrument with sufficient collecting area and CR-background rejection power to measure the IGRB at energies $>100 \mathrm{GeV}$. Since launch into lowEarth orbit on 2008 June 11, the LAT has operated primarily in a sky-survey mode that, combined with a large field of view $(2.4 \mathrm{sr})$ and good spatial resolution $\left(\sim 1^{\circ}\right.$ at $\left.1 \mathrm{GeV}\right)$, has enabled the most detailed studies of the DGE to date. The LAT is a pair-conversion telescope consisting of a precision tracker and imaging calorimeter, which are used together to reconstruct $\gamma$-ray directions and energies, and a surrounding segmented anticoincidence detector (ACD) to identify charged particles entering the instrument. Atwood et al. (2009) provide a description of the Fermi mission and LAT detector, as well as details of the on-orbit calibration. Ground data processing, event selection, and instrument response functions (IRFs) are provided in Abdo et al. (2009c), Ackermann et al. (2012b, 2012d).

A first measurement of the IGRB spectrum between $200 \mathrm{MeV}$ and $100 \mathrm{GeV}$ based on 10 months of LAT data was published in Abdo et al. (2010b). In this paper we present a refinement and extension of that analysis based on 50 months of sky-survey observations. Multiple improvements in event classification, Galactic foreground and CR background models, and analysis techniques have been implemented. Together with increased statistics, these updates allow for an extension of the LAT IGRB measurement by over a decade in energy, now covering the range from $100 \mathrm{MeV}$ to $820 \mathrm{GeV}$.

\section{DATA SAMPLES}

Fifty months of LAT data recorded between 2008 August 5 and 2012 October 6 are used for this analysis, corresponding to a total observation time of 1239 days. ${ }^{71}$ The events have been reprocessed with an updated instrument calibration, which improves the agreement between data and simulation of the energy reconstruction quality, the point-spread function (PSF), and certain classification variables and thereby reduces systematic uncertainties (Bregeon et al. 2013). ${ }^{72}$

The LAT IGRB analysis poses especially stringent requirements on the $\gamma$-ray purity of the event selection since both the signal and CR-background spatial distributions are quasiisotropic. The residual CR background contamination must be reduced to a relatively small fraction of the total isotropic intensity in order to measure the IGRB with acceptable systematic uncertainty because the (not perfectly known) CR background is directly subtracted from the total isotropic intensity in the final step of evaluating the IGRB.

The predefined event classes publicly available from the Fermi Science Support Center, including P7ULTRACLEAN, have insufficient $\mathrm{CR}$ background rejection performance for the IGRB analysis energies below $E<400 \mathrm{MeV}$ and energies above $E>100 \mathrm{GeV}$. Therefore, we developed two dedicated event samples for the IGRB analysis with distinct selection criteria at low and high energies. The IGRB intensity measurements reported in Section 5 use the "low-energy" sample for the energy range $100 \mathrm{MeV}$ to $13 \mathrm{GeV}$ and the "high-energy" sample for the energy range $13-820 \mathrm{GeV} .^{73}$ Multiple event classifications are necessary in order to obtain the best-possible compromise between statistics and low CR backgrounds across the full LAT energy range since the compositions and interactions of $\mathrm{CR}$

\footnotetext{
71 LAT data recording is disabled for $\approx 13 \%$ of the total on-orbit time, during passages through the South Atlantic Anomaly (SAA), a region with extremely high charged particle backgrounds. Only observation periods that passed data quality monitoring and where the angle between the LAT $z$-axis and the zenith was below $52^{\circ}$ are used for this analysis. Note that the actual live time is $\sim 8 \%$ smaller than the 1239 day observation time quoted here owing to instrumental dead time associated with event latching and readout.

72 The reprocessed data are available from the Fermi Science Support Center (http://fermi.gsfc.nasa.gov/ssc/), together with a list of caveats regarding their usage.

73 Each sample includes events from the full LAT energy range. The labels "low-energy" and "high-energy" refer to the energy regime for which the event classifications have been optimized. The energy overlap between samples allows for consistency checking between the low-energy and high-energy analyses (described in Section 3), a feature we used to verify that the specific choice of crossover energy between 5 and $50 \mathrm{GeV}$ does not affect the accuracy of our quoted results for the IGRB intensity.
} 
Table 1

Event Selection Criteria for Low-energy and High-energy Samples, Including Modifications with Respect to P7ULTRACLEAN

\begin{tabular}{lcc}
\hline \hline & Low-energy & High-energy \\
\hline Data Sample/Event Selection & P7REP_IGRB_LO & P7REP_IGRB_HI \\
\hline Add tracker veto I & $\mathrm{Y}$ & $\mathrm{Y}$ \\
Add tracker veto II & $\mathrm{Y}$ & $\mathrm{N}$ \\
Add deposited charge veto & $\mathrm{Y}$ & $\mathrm{N}$ \\
Remove calorimeter shower maximum veto & $\mathrm{N}$ & $\mathrm{Y}$ \\
Incidence angle veto & $>72^{\circ}$ & $>72^{\circ}$ \\
Zenith angle veto & $>90^{\circ}$ & $>105^{\circ}$ \\
\hline
\end{tabular}

Notes. See Section 2.1 for detailed descriptions of the event selection criteria. The low-energy and high-energy event samples are used to derive the IGRB intensity in the $100 \mathrm{MeV}$ to $13 \mathrm{GeV}$ and $13 \mathrm{GeV}-820 \mathrm{GeV}$ energy ranges, respectively.

backgrounds in the low- and high-energy regimes are rather different. The modifications to the baseline P7ULTRACLEAN classification for the two event samples used in this work are described below and summarized in Table 1 .

\subsection{Event Selection}

The low-energy sample is a strict subset of events classified as photons according to the P7ULTRACLEAN event class definition (Ackermann et al. 2012b). To reduce the residual background of secondary electrons, positrons, and protons produced by CR interactions in the Earth's atmosphere, which are the primary concern in the low-energy IGRB analysis, the following additional criteria are imposed.

Tracker veto I. Part of the tracker is used as an additional veto to complement the ACD. Specifically, we require the reconstructed $\gamma$-ray trajectory to cross at least two layers of active silicon strip detector area without producing hits in these detectors. This selection criterion significantly increases the efficiency of vetoing charged particles entering the LAT.

Tracker veto II. We discard events for which the reconstructed pair-conversion vertex lies in the three $x-y$ double layers of the tracker closest to the calorimeter. Comparisons of lowbackground and high-background on-orbit data sets, as well as comparisons of $\gamma$-ray and CR-background Monte Carlo simulations, have shown that these events suffer a higher background contamination fraction.

Deposited charge veto. $\gamma$-rays convert into an electron-positron pair, while most of the background events involve a single charged particle. Therefore, we require the charge deposited in the first tracker layer following the interaction vertex to be $>1.5$ times the value expected for a minimum ionizing particle, which is typically indicative that two particles crossed the layer rather than one by itself.

Incidence angle veto. Events arriving from directions $>72^{\circ}$ off the LAT boresight are rejected because there is increased CR background leakage for such highly inclined events.

The new event class for the low-energy sample is denoted as P7REP_IGRB_LO in the remainder of this manuscript to distinguish it from the publicly available standard event classes. The sky-averaged exposure of the P7REP_IGRB_LO selection is $66 \%$ of the exposure of the corresponding P7ULTRACLEAN selection for survey mode observations (see Figure 1), when compared at the energy of maximum exposure. The estimated residual CR background rate is reduced by a factor of $\sim 3$ around $200 \mathrm{MeV}$, where the background rate is highest.

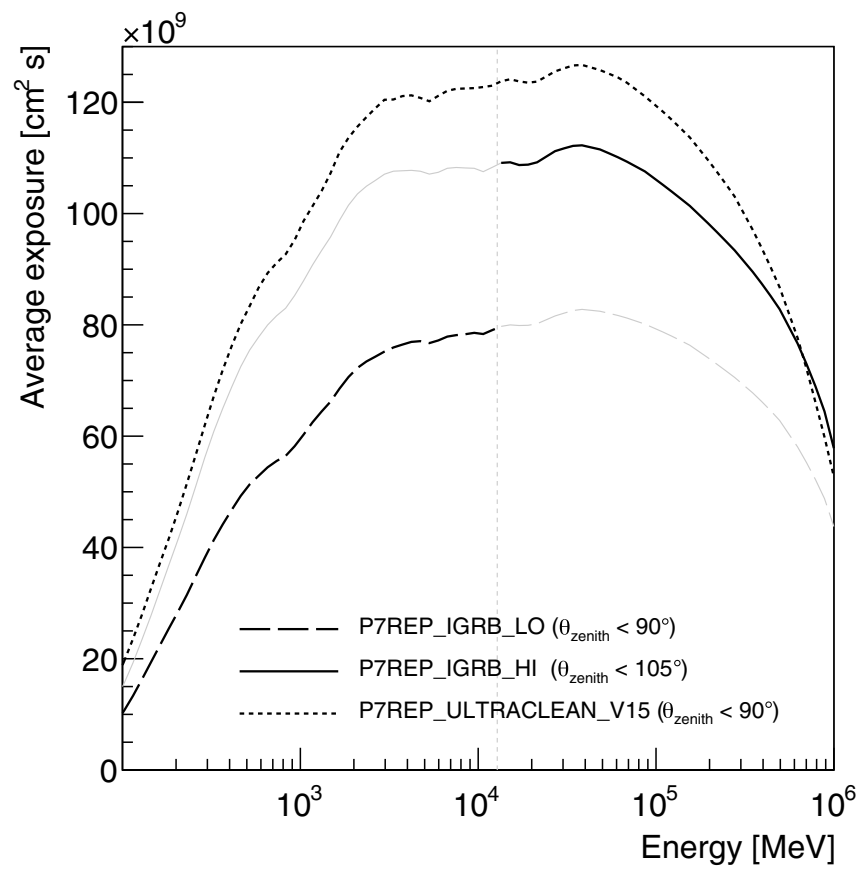

Figure 1. Comparison of the sky-averaged exposure for the P7REP_IGRB_LO, P7REP_IGRB_HI, and P7ULTRACLEAN event selections. Thick lines indicate the respective energy ranges for which the P7REP_IGRB_LO and P7REP_IGRB_HI event classes are used in this analysis.

As a final step to define the low-energy sample, events from measured directions $>90^{\circ}$ off the Earth zenith are rejected to limit contamination by photons from the Earth limb (Abdo et al. 2009a).

For the high-energy sample, we use a relaxed event selection compared to P7REP_IGRB_LO. At energies above $13 \mathrm{GeV}, \mathrm{CR}$ primaries in the form of protons and heavier nuclei dominate the background flux. The rejection power for $\mathrm{CR}$ nuclei is sufficient for this analysis if one requires only the condition described above as "Tracker veto I" in addition to the standard P7ULTRACLEAN event class definitions. We implement the "Incidence angle veto" as for the low-energy event class.

The standard P7ULTRACLEAN event classification rejects events for which the positions of the primary interaction vertex and the reconstructed shower maximum are separated by $>12$ radiation lengths as measured along the shower axis. This selection criterion was introduced to reject CR events with bad shower reconstructions that would sometimes result in large apparent depths for the shower maxima-a strategy that works well for energies $\lesssim 500 \mathrm{GeV}$ but removes a significant fraction 
of $\gamma$ rays $\gtrsim 500 \mathrm{GeV}$. Therefore, this selection criterion is removed in the event selection for the high-energy sample. The very moderate increase in residual $\mathrm{CR}$ background arising from this removal is overcompensated by the "Tracker veto I" condition that was introduced for this event class.

The distinct classification scheme for the high-energy sample is denoted as P7REP_IGRB_HI in contrast to the P7REP_IGRB_LO classification used for the low-energy sample. At high energies, the reconstructed arrival directions of CRinduced atmospheric $\gamma$ rays are confined to angles very close to the Earth limb ( $113^{\circ}$ from the Earth zenith) owing to the reduced width of the PSF ( $\sim 0.1$ above $10 \mathrm{GeV}$ ). Therefore, the zenith angle veto condition described above for the low-energy sample is modified to reject only photons from directions $>105^{\circ}$ off the Earth zenith.

The P7REP_IGRB_HI selection has a peak exposure of about $85 \%$ of the peak exposure of the standard P7ULTRACLEAN selection, and it surpasses the P7ULTRACLEAN selection in acceptance $\gtrsim 700 \mathrm{GeV}$.

Both new event selections were cross-validated against the standard P7ULTRACLEAN event selection by performing the analysis described below also on the P7ULTRACLEAN data set. We obtain consistent results in the energy range in which we can perform the analysis even in the presence of the higher CR background of the P7ULTRACLEAN selection (400 MeV to $100 \mathrm{GeV}$ ).

\subsection{Instrument Response Functions}

New sets of dedicated IRFs were created for the low-energy (P7REP_IGRB_LO) and the high-energy (P7REP_IGRB_HI) event classes via Monte Carlo simulation of $\gamma$-rays. The energy range of the new IRFs is $17.8 \mathrm{MeV}$ to $1.78 \mathrm{TeV}$.

Figure 1 shows the sky-averaged exposures obtained for the low-energy and high-energy samples using the corresponding P7REP_IGRB_LO and P7REP_IGRB_HI IRFs, respectively. The exposure that would be obtained for the same observation period, but using the standard P7_ULTRACLEAN event sample with IRFs P7REP_ULTRACLEAN_V15, is plotted for comparison. For the P7REP_IGRB_HI selection, there is an overall drop in exposure as a result of using part of the silicon tracker to veto charged particles. However, at the highest energies, especially $>300 \mathrm{GeV}$, this loss is increasingly counteracted by the removed shower maximum constraint in the P7REP_IGRB_HI class (see Section 2.1). The P7REP_IGRB_LO selection has a significantly lower average exposure than P7REP_IGRB_HI. This loss of exposure is acceptable at low energies where this event class is used since the IGRB measurement is not limited by statistics below tens of $\mathrm{GeV}$.

In-flight PSF corrections available for the IRFs corresponding to standard event classes have not been applied to the P7REP_IGRB_LO and P7REP_IGRB_HI IRFs. The corrections were motivated by small differences observed in the PSF of the original (P7) on-orbit and simulated LAT data at energies $\gtrsim 1 \mathrm{GeV}$ (Ackermann et al. 2012b, 2013a). We verified directly that such small corrections, mitigated in the reprocessed data (Bregeon et al. 2013), do not significantly affect this analysis. This is expected since it is performed on a spatial grid of about 0.9 , considerably larger than the typical high-energy PSF.

\subsection{Residual Cosmic-ray Background}

Charged and neutral CRs misclassified as $\gamma$-rays by the multivariate event classification algorithms mimic an isotropic flux that is indistinguishable from the IGRB. In addition, genuine $\gamma$-rays from the Earth's atmosphere that have directional reconstruction errors sufficient to bypass the zenith angle veto become a source of apparent extraterrestrial emission over the full sky. In this work, the term "CR background" includes CR-induced $\gamma$-rays from the atmosphere.

Our estimation of the residual CR background event rate is based on Monte Carlo simulations of the relevant particle species in the near-Earth environment, namely, CR nuclei and electrons, as well as their atmospheric secondaries. We simulate both CR backgrounds and signal $\gamma$-rays and extract the distributions for reconstructed event properties with the greatest discrimination power at low and high energies, respectively: the multivariate event classifier output and the transverse shower size. The distributions for simulated background and signal are compared to the distributions for the flight data to quantify the level and associated uncertainty of the CR background. A detailed description of this method can be found in Ackermann et al. (2012b).

To account for atmospheric $\gamma$-rays surviving the zenith angle veto, an updated phenomenological model for the Earth emission based on LAT observations is included in the Monte Carlo simulation. Atmospheric $\gamma$-rays can bypass the zenith veto either by being reconstructed in the extreme tail of the PSF or by entering from the back side of the instrument and being reconstructed as though coming from the front. Although such catastrophic mis-reconstructions are rare, the Earth emission is sufficiently bright that the expected event rate is nonnegligible at energies $\lesssim 1 \mathrm{GeV}$ (Bechtol 2012). For the stringent zenith angle selections used in this work, the residual contamination of atmospheric $\gamma$-rays is expected to be composed primarily of back-entering events. The reconstructed directional distribution of back-entering atmospheric $\gamma$-rays in particular is approximately isotropic.

Figure 2 shows the residual $\mathrm{CR}$ background rates as a function of reconstructed energy for the P7REP_IGRB_LO and P7REP_IGRB_HI classes. Note that the event energy is reconstructed under the hypothesis of a front-entering $\gamma$-ray and in general does not represent the actual energy for hadrons. At high energies, primary protons and electrons both contribute significantly to the CR background. Although protons are far more abundant than electrons in the environment of the LAT, there is also greater rejection power against protons since analysis of the shower shape in the calorimeter can be used to tag and remove protons in addition to the veto power obtained from the ACD. All contributions shown in Figure 2 have been adjusted from the raw Monte Carlo predictions based on event property comparisons between the simulated and flight data, as described above. The CR background contamination after rescaling agrees with the raw predictions from Monte Carlo simulation to within 35\%, depending on energy, and this maximum discrepancy is used as a measure of the systematic uncertainty in the CR background contamination.

The full uncertainty in residual CR background rates, shown in Figure 2, has been calculated by adding systematic and statistical uncertainties in quadrature. For the P7REP_IGRB_HI event class at energies above $10 \mathrm{GeV}$ (relevant for the highenergy sample), the statistical uncertainties are large owing to the limited size of the simulated residual background sample. ${ }^{74}$ Therefore, instead of using bin-by-bin estimates for the CR

\footnotetext{
74 The existing background rate estimates were derived from several million CPU hours of CR simulation. Significant gains in precision might be achieved in the future when more computing power becomes available.
} 


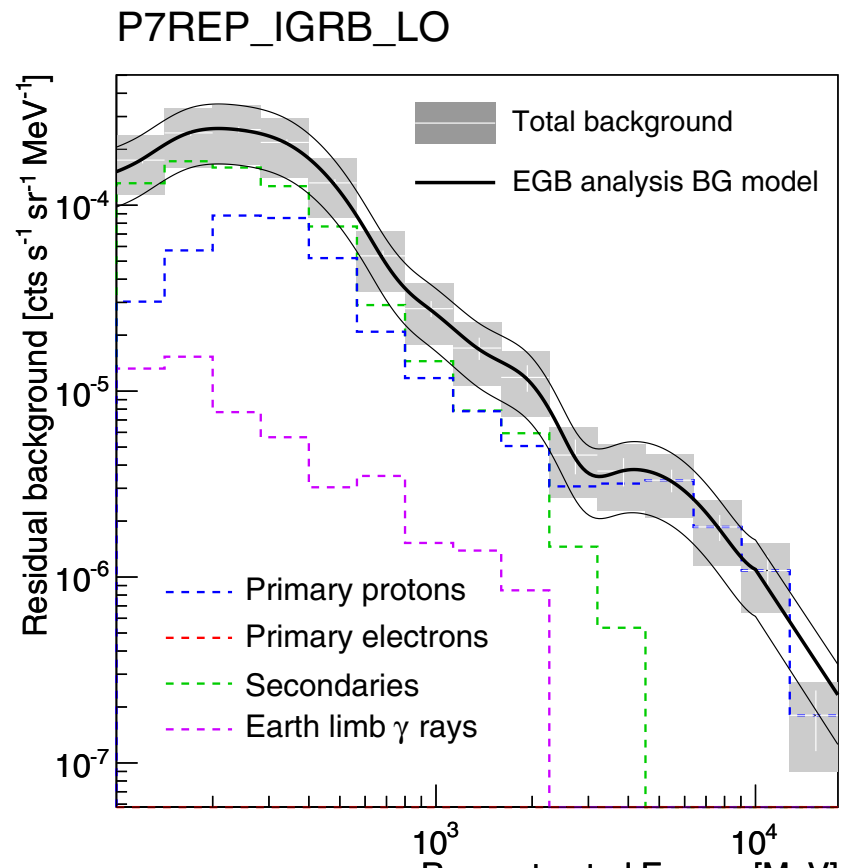

Reconstructed Energy [MeV]

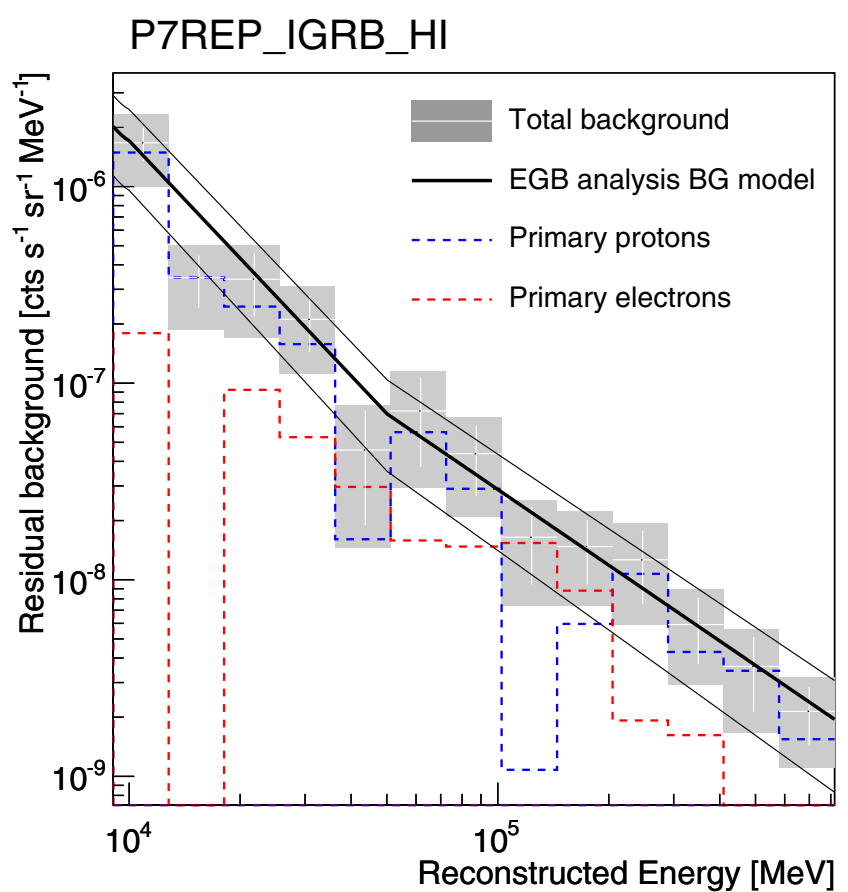

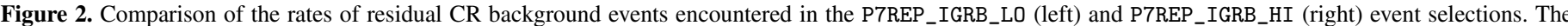

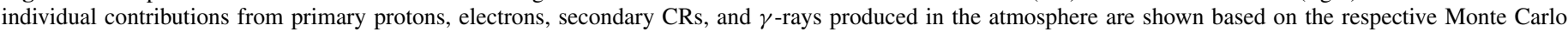

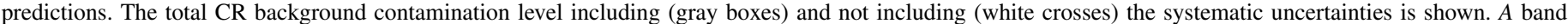
consisting of three black lines displays the model that is used for the level (thick line) and the uncertainty (thin lines) of the CR background in the IGRB analysis.

background rates in the high-energy IGRB analysis, the rates are obtained from a fit to the (rescaled) simulated background rates between 10 and $820 \mathrm{GeV}$ using a simple broken power-law (PL) function in energy with a break at $50 \mathrm{GeV}$. A spline interpolation of the background rates is used as the CR background model below $10 \mathrm{GeV}$.

\section{DERIVATION OF THE IGRB SPECTRUM}

The spectrum of the IGRB is derived in a two-step procedure. First, the spectrum of the isotropic component is determined as part of a multicomponent maximum likelihood fit of template maps to the observed LAT counts using the tools and method described in Ackermann et al. (2009). This isotropic component is attributed to the sum of the IGRB and misclassified CR backgrounds (Section 2.3). Second, the CR background contribution is subtracted from the isotropic component to obtain the IGRB spectrum.

In the multicomponent maximum likelihood fit, we create template maps containing the number of LAT counts expected during the observation period for various contributions to the $\gamma$ ray sky. Each template map is based on a model or measurements of the respective contribution. The $\gamma$-ray emission observed by the LAT is modeled using five template maps in addition to the isotropic emission and 403 point sources that are fitted individually. Two template maps describe the DGE. One map is used for the solar disk and inverse Compton (IC) emission associated with the solar radiation field. One map describes the local diffuse emission from Loop I and the Local Loop. A last map is used to describe contributions from established point sources that are not individually fitted.

The normalization of each template is fitted individually for each energy bin in the energy range between $100 \mathrm{MeV}$ and $13 \mathrm{GeV}$ using the low-energy event sample. This fit is performed by maximizing the likelihood to obtain the observed number of counts in each pixel given our model. We denote this fit result as the "low-energy fit." Above $13 \mathrm{GeV}$, the normalizations of the Galactic foreground templates are kept fixed over the full highenergy range, i.e., we use the spectral shape that is provided in the templates to model the foreground in addition to the spatial information. Only the normalizations of the isotropic template and the point sources are fitted for each individual energy bin above $13 \mathrm{GeV}$. To determine the fixed template normalization factors, we first fit the normalization of each Galactic foreground template in the six energy bins between 6.4 and $51 \mathrm{GeV}$ using the same procedure as for the low-energy fit (the number of events above $51 \mathrm{GeV}$ is too low to robustly fit all foregrounds individually in each energy bin). For each respective foreground template, the average normalization factor from these six energy bins is applied to all of the energy bins between 13 and $820 \mathrm{GeV}$. The bin-by-bin fitting and average-value scaling procedures yield consistent spectral forms in the $6.4-51 \mathrm{GeV}$ range (see Figures 14, 16, and 18 in Appendix A), providing confidence to the extrapolation above $51 \mathrm{GeV}$ based on the spectral model shape of each foreground component. Moreover, the shapes of the high-energy spectra of the dominant Galactic foreground contributions (i.e., the interactions of CRs with ISG and ISRF) can be derived quite robustly based on the well-measured local $\mathrm{CR}$ electron and nucleon spectra. Modeling uncertainties are therefore small.

After the Galactic foreground template normalizations are fixed to these average values, a multicomponent maximum likelihood fit is performed using the high-energy event sample in the energy range between 13 and $820 \mathrm{GeV}$, which we denote as the "high-energy fit."

The template maps are binned on a HEALPix ${ }^{75}$ grid of order $6(\approx 0.9$ pixel size; Górski et al. 2005) in Galactic longitude and

\footnotetext{
75 http://healpix.jpl.nasa.gov/healpixSoftwareGetHealpix.shtml
} 
latitude, and in 26 energy bins between $100 \mathrm{MeV}$ and $820 \mathrm{GeV}$. Galactic diffuse emission dominates the intensity of the $\gamma$-ray sky at low Galactic latitudes. This emission originates from the interactions of CRs with ISG and the ISRF. In the former case, $\gamma$-rays are produced through the generation and decay of neutral pions, or through nonthermal bremsstrahlung; in the latter case, $\gamma$-rays are produced by the IC process. We consider the $\gamma$-ray emission due to interactions with ISG and the ISRF separately in this analysis. The spatial distribution of $\gamma$-rays from interactions with ISG is well correlated to the column density of ISG along a given line of sight, whereas $\gamma$-rays created through interactions with the ISRF form a comparatively smooth emission component peaked at the Galactic center. We use the GALPROP ${ }^{76}$ code (Strong et al. 2000; Vladimirov et al. 2011) to obtain templates for these two components. A detailed discussion of the modeling of the Galactic diffuse emission will be presented in Section 4 and Appendix A. We refer to the two template maps as the "H I + H II" template and the "inverse Compton" template below.

The second prominent contribution to the $\gamma$-ray sky is from the individually resolved LAT $\gamma$-ray sources. We include the 403 sources from the second LAT source catalog (2FGL; Nolan et al. 2012) from above and below the Galactic plane $\left(|b|>2^{\circ}\right)$ that are detected with a test statistic (TS) larger than 200 in that catalog as individual templates. The 1215 sources with TS values less than 200 are added to a common template using the spectral information found in the 2FGL catalog. Additionally, we add a template for a source at the position of CRATES J231012-051421 (Healey et al. 2007) after a localized excess in the residual map was found in a first iteration of this analysis at a position consistent with the CRATES source. As a result of the difference in the observation time between this work and the 2FGL catalog (50 months versus 24 months) and the intrinsic variability of many high-latitude LAT sources, both extra sources and changes in their time-averaged spectra can be expected. However, no systematic search for additional sources too faint to be identified on the residual map was performed on the data samples used in this analysis. We note that as a result of the considerably more stringent event selection used in this work in comparison to the 2FGL catalog, the effective gain in exposure is much smaller than what is indicated by the difference in observation length. The threshold in $\gamma$-ray flux for detecting sources in this sample would only be marginally lower than the flux threshold for the source sample in the 2FGL catalog.

A third contribution to the $\gamma$-ray sky that is included as a component in the likelihood fit is the $\gamma$-ray emission related to the Sun. Gamma rays are produced by CR collisions with the outer atmosphere of the Sun and by IC scattering of CR electrons off the solar radiation field. The solar disk and extended IC emission have been measured using the LAT (Abdo et al. 2011). We use the Solar System Tools (Johannesson \& Orlando 2013) from the LAT ScienceTools (v9r30p0) to create a template for the time-averaged solar emission in our observation period that is based on this measurement. This template is denoted as "Solar Disk + IC" throughout this work. To avoid bias at high energies, where the solar spectrum is not well known by measurements, we do not use the $\pm 3^{\circ}$ region around the ecliptic plane in the fit for energies above $13 \mathrm{GeV}$ (in the energy range where the highenergy sample is used). The $\gamma$-ray emission from the Moon (Abdo et al. 2012) has been neglected in this work since the

\footnotetext{
76 http://galprop.stanford.edu
}

Moon does not feature an extended IC contribution that could bias a measurement of the IGRB.

Structures are seen in the diffuse $\gamma$-ray emission that are correlated to Loop I (seen most prominently in the region of the North Polar Spur; Casandjian \& Grenier 2009). Loop I is also bright in the $408 \mathrm{MHz}$ radio continuum survey of Haslam et al. (1982), indicating a local overdensity of highenergy electrons or stronger magnetic fields in that region. A detailed investigation of the spectrum and spatial distribution of the $\gamma$-ray emission from this region has not yet been performed but is outside the scope of this paper. We therefore use a simple geometrical model (Wolleben 2007) for the synchrotron emission from Loop I and the Local Loop to generate a template for the $\gamma$-ray emission from these structures (referred to as the "Loop I/Local Loop" template).

Systematic uncertainties associated with the foreground templates mentioned above and other foreground modeling choices, e.g., the optional inclusion of an additional template for the Fermi bubbles, are discussed in Section 5.2.

Certain regions in the vicinity of the Galactic plane have been masked, and the corresponding pixels have not been used in the likelihood fit. The shape of the mask has been chosen to reduce systematic uncertainties connected to the Galactic diffuse foreground emission by excluding regions in which the column density of the ISG is not dominated by the atomic and ionized hydrogen in the vicinity of our solar system. Details of the definition of the mask are listed in Appendix C. Figure 3 shows the integrated LAT counts above $100 \mathrm{MeV}$ that are used for this analysis and the excluded regions.

\section{FOREGROUND DIFFUSE GALACTIC EMISSION MODELS}

At high Galactic latitudes $\left(|b| \gtrsim 10^{\circ}\right)$ the ISG is dominated by atomic gas clouds within a few hundred parsecs, a range in which we do not expect a significant change in the density of the CRs that interact with these clouds (Ackermann et al. 2013b). Therefore, the spectrum and spatial distribution of the $\gamma$-rays arising from the $\mathrm{CR}$ interactions with atomic gas are relatively well constrained by measurements of the gas cloud distributions and direct measurements of local CR spectra. The proximity of ISG seen at high Galactic latitudes permits the use of a single model template as opposed to multiple templates describing the ISG at various distances, as is typical for other DGE studies. Only a small fraction of the ISG-related $\gamma$-ray emission arises from ionized hydrogen gas, which has a larger scale height than the atomic gas and a less well known distribution on the sky.

The spectrum and intensity distribution of the IC-related $\gamma$-ray emission on the sky can only be predicted from a global modeling of CR propagation and interaction in the Galaxy. It is highly dependent on the injection and propagation of electrons in the Galactic plane and into the Galactic halo. It further depends on the spatially varying spectrum of the ISRF, which is more uncertain than the distribution of the ISG.

\subsection{Reference Foreground Models}

An extensive study comparing LAT data to GALPROP-based predictions of the DGE has been published in Ackermann et al. (2012g). However, that study was restricted to a selected set of propagation and CR injection scenarios. In particular, it was assumed that the diffusion coefficient is constant throughout the Galaxy and the source population that injects the electrons 


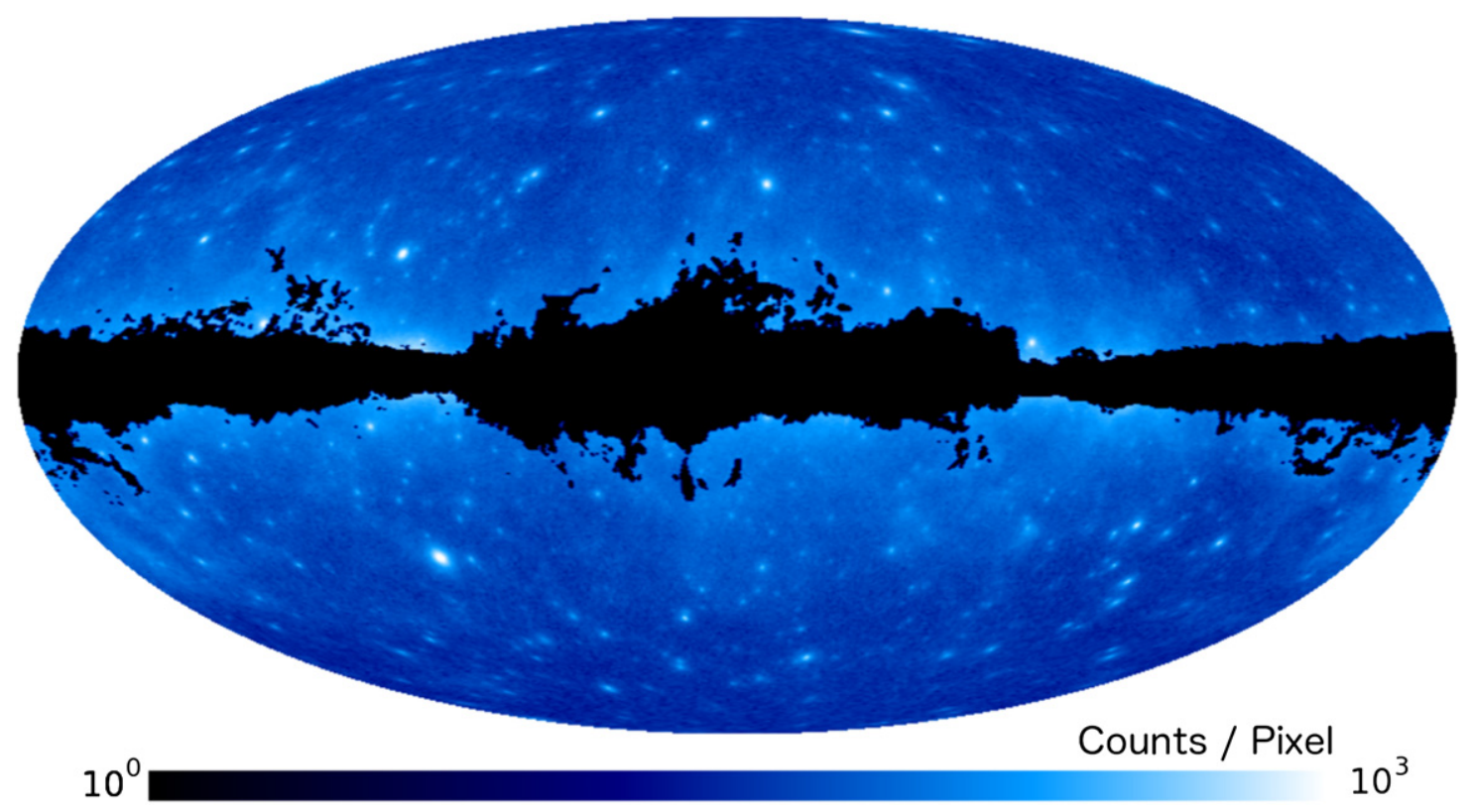

Figure 3. Map of counts observed by the Fermi LAT above $100 \mathrm{MeV}$ using a Mollweide projection in Galactic coordinates with a pixel scale of $\approx 0.9$. The color scale is logarithmic. Overlaid is the mask used in this analysis to exclude regions from the template-fitting procedure (see Appendix C for details).

Table 2

Comparison of Benchmark Galactic Foreground Models

\begin{tabular}{ll}
\hline \hline Foreground & \multicolumn{1}{c}{ Main features and differences with respect to other DGE models } \\
\hline Model A & $\begin{array}{l}\text { Sources of CR nuclei and electrons trace pulsar distribution; } \\
\text { constant CR diffusion coefficient and reacceleration strength through Galaxy }\end{array}$ \\
\hline Model B & $\begin{array}{l}\text { Additional electron-only source population near Galactic center, } \\
\text { these electrons are responsible for majority of IC emission; } \\
\text { local source of soft CR electrons needed to explain CR electron spectrum at Earth below 20 GV }\end{array}$ \\
\hline Model C & $\begin{array}{l}\text { Sources of CR nuclei and electrons more centrally peaked than pulsar distribution; } \\
\text { CR diffusion coefficient and reacceleration strength vary with galactocentric radius and height }\end{array}$ \\
\hline
\end{tabular}

Notes. See Appendix A for a more detailed description of these three reference DGE models.

is the same as the source population that injects the nuclei. These choices are well motivated by Occam's razor, but we demonstrate in Appendix A that template maps for the IC emission that are derived from DGE models of this type lead to inconsistencies when used in our multicomponent likelihood fit. For example, the spectrum of the IC emission predicted by the model does not match the spectrum obtained in the fit to the LAT data. Such a mismatch is critical for the IGRB study if it originates from an inaccurate model of the IC intensity distribution on the sky. In this case, the isotropic template could partially compensate for the inaccuracies of the IC intensity distribution and thereby lead to a biased IGRB measurement.

We therefore extend the study of foreground models to include two reference models for propagation and injection scenarios with more degrees of freedom than those considered in Ackermann et al. (2012g). One allows for distinct populations injecting CR electrons and nuclei. The other allows a variation of the diffusion coefficient with radial distance from the Galactic center and height above the Galactic plane. These two reference models are described in more detail in Appendix A. We denote them as foreground models " $\mathrm{B}$ " and " $\mathrm{C}$ ", respectively, to distinguish them from foreground model " $A$ " that is derived from the class of DGE models studied in Ackermann et al. $(2012 \mathrm{~g})$. The principal features of and differences between the three reference foreground models are summarized in Table 2.
Our main concern in this work is to investigate whether the fitted spectral features of the IGRB depend on the specific type of foreground model chosen. It is not the aim or scope of this work to perform a quantitative study of whether one of the alternative foreground model classes matches the LAT data better than another. For simplicity, we use model A as a baseline for the purpose of quoting certain results and testing variations of DGE model parameters. However, we do not view model A as canonical or preferred over the other models.

All three foreground models assume diffusive CR transport with reacceleration in the interstellar medium (ISM). The diffusion coefficient has a PL dependence on rigidity with index $\delta=0.33$, as expected from a Kolmogorov spectrum of magnetic turbulences. CR propagation and injection parameters within each model are chosen to obtain good agreement between the predicted local spectra of interstellar CRs and actual CR measurements after solar modulation effects have been taken into account. In particular, we require good agreement with the measured proton and helium spectra, the electron spectrum, and the $\mathrm{B} / \mathrm{C}$ and ${ }^{10} \mathrm{Be} /{ }^{9} \mathrm{Be}$ ratios. ${ }^{77}$

\footnotetext{
77 One notable exception is the electron spectrum for foreground model B that is tuned to reproduce the observed IC emission spectrum rather than the measured CR electron spectrum. A detailed description of the various injection and propagation parameters for all three foreground models can be found in Appendix A.
} 
The distribution of $\mathrm{H}_{2}$ and $\mathrm{H}$ I gas in the Galaxy is modeled based on the microwave survey of Dame et al. (2001) and the radio survey of Kalberla et al. (2005), respectively (see also Appendix C). Details regarding the gas distribution modeling can be found in Ackermann et al. (2012g). An analytic model is used for the distribution of ionized hydrogen in the Galaxy (Gaensler et al. 2008). We use the ISRF model introduced in Porter et al. (2008), which is available within GALPROP. We take into account the anisotropy of the ISRF by calculating for 192 uniformly distributed lines of sight the ratio between the predicted IC emission from a full anisotropic calculation and the prediction assuming that the ISRF is isotropic. This set of ratios is then interpolated and applied as a multiplicative correction to all the generated IC templates.

It has been shown that interstellar dust can trace gas that is not seen in H I or CO surveys (Grenier et al. 2005). Therefore, we use the $E(B-V)$ visual reddening map provided by Schlegel et al. (1998), a tracer of the interstellar dust column density, to estimate the total ISG column density along a line of sight. We use the procedure described in Ackermann et al. (2012g) to obtain a conversion factor between the magnitude of reddening in the $E(B-V)$ map and the H I gas column density (denoted as $\mathrm{HI}$-to-dust ratio below). The procedure uses a linear regression between the $E(B-V)$ map and the $H_{\mathrm{I}}$ and $\mathrm{CO}$ surveys to obtain the $\mathrm{H}$ I-to-dust ratio in regions of the sky where $E(B-V)<5$ mag. The fit depends slightly on the spin temperature $T_{S}$ that one assumes to correct for the opacity of the $21 \mathrm{~cm}$ line in the H I surveys. We find an $\mathrm{HI}$-to-dust ratio of $7.9 \times 10^{21} \mathrm{~cm}^{-2} \mathrm{mag}^{-1}$ assuming the widely used value of $T_{S}=125 \mathrm{~K}$ for the spin temperature (e.g., Kulkarni \& Heiles 1988), and we use this H I-to-dust ratio in our analysis. Note that this value is different from the value used in Ackermann et al. (2012g), where the higher spin temperature of $T_{S}=150 \mathrm{~K}$ was used for deriving this conversion factor. The lower spin temperature used here leads to smaller residuals between the $E(B-V)$ and the $\mathrm{H}$ I and $\mathrm{CO}$ survey derived gas column densities at high Galactic latitudes that are relevant for the IGRB analysis.

\subsection{Additional Foreground Models Used for Systematics Studies}

In addition to our three reference foreground models, we consider further variations of foreground models to assess the systematic uncertainties for the derivation of the IGRB that are related to the modeling of the DGE. Specifically, we study variations of the size of the CR halo between 4 and $10 \mathrm{kpc}$, a variation of the distribution of $\mathrm{CR}$ sources in galactocentric radius, a model where CRs are not reaccelerated in the Galaxy, models with an extra foreground template for the Fermi bubbles, a higher radiation field in the Galactic bulge, a lower random Galactic magnetic field than in the default models, and a variation of the $\mathrm{H}$ I-to-dust ratio by $10 \%$.

Foreground model A serves as the baseline model for these variations. To assess the stability of the IGRB measurement with respect to assumptions regarding the halo size and the CR source distribution, we use three models discussed in Ackermann et al. (2012g) chosen to cover the extreme values for the CR halo size (4 kpc versus $10 \mathrm{kpc}$ ) and radial source distribution (traced by pulsars versus traced by supernova remnants, SNRs) in the range of models studied there.

Strong et al. (2011) suggest that CR propagation models where CRs are not reaccelerated in the Galaxy (so-called plain diffusion models) describe the synchrotron emission observed from the Galaxy better than present models with reacceleration.
We therefore include such a plain diffusion model in our investigations.

Dobler et al. (2010) and $\mathrm{Su}$ et al. (2010) have noted the existence of large-scale structures of residual diffuse $\gamma$-ray emission above and below the Galactic center region that became well known as the "Fermi bubbles." Although well established as significant features, the bubbles were not included in the reference foreground models. The Fermi bubbles have been studied exclusively as a residual after subtracting a model of the DGE and isotropic diffuse emission. No template for their shape has yet been derived from independent observations. Using a template derived from a strictly empirical excess of $\gamma$ ray emission might lead to a bias in the other components of the fit, including the isotropic template. Since neglecting the emission from the Fermi bubbles might bias the fit as well, we tested the effects of including template maps for the Fermi bubbles in the multicomponent fit. Two models for the intensity distribution of the Fermi bubbles on the sky were tested. The first is a simple geometric template used for the investigation of systematic uncertainties in studies of the $\gamma$-ray emission from SNRs (de Palma et al. 2013). The second is a template derived from the residual $\gamma$-ray emission (Fermi LAT Collaboration 2014).

Our knowledge of the ISRF in the inner Galaxy is limited. We therefore repeat the IGRB fit against an ISRF model that assumes a factor of 10 higher stellar luminosity in the Galactic bulge. Such a model is still compatible with constraints derived from observations of the ISRF in the solar neighborhood.

We also test the impact of the assumed random magnetic field strength by generating a foreground model with a lower random magnetic field strength of $3 \mu \mathrm{G}$ in the solar neighborhood, to compare with the reference models $\mathrm{A}$ and $\mathrm{B}$, which use a value of $7.5 \mu \mathrm{G}$ for the random magnetic field there (see in this context Appendix A.1).

The difference in the $\mathrm{HI}$-to-dust ratio between the spin temperature value of $T_{S}=150 \mathrm{~K}$ adopted in Ackermann et al. (2012g) and the widely used spin temperature value of $T_{S}=125 \mathrm{~K}$ that was used in this work is of the order of $10 \%$. We test foreground models with variations of the H I-to-dust ratios by $\pm 10 \%$ to determine the impact of this parameter on the IGRB measurement.

The impact of all described variations in modeling the foreground DGE on the spectrum of the IGRB is discussed in Section 5.2, together with a model-independent study to assess the impact of unmodeled residuals in the foreground emission.

\section{RESULTS}

\subsection{IGRB Spectrum}

The likelihood fitting technique introduced in Section 3 is used to derive the spectrum of the isotropic emission for the three different DGE foreground models described in Section 4. The residual particle background contamination is subtracted from the isotropic component to obtain the spectrum of the IGRB.

Figures 4-6 show the results of the fits using foreground models A, B, and C, respectively. Each figure displays the average high-latitude $\left(|b|>20^{\circ}\right)$ intensities attributed to the isotropic emission (IGRB plus CR background), the individual sources, two DGE components, the solar emission, and the local foreground templates. The sum of these intensities is compared to the average $\gamma$-ray intensity observed by the LAT. A separate graph shows the contributions of the IGRB and of the residual 

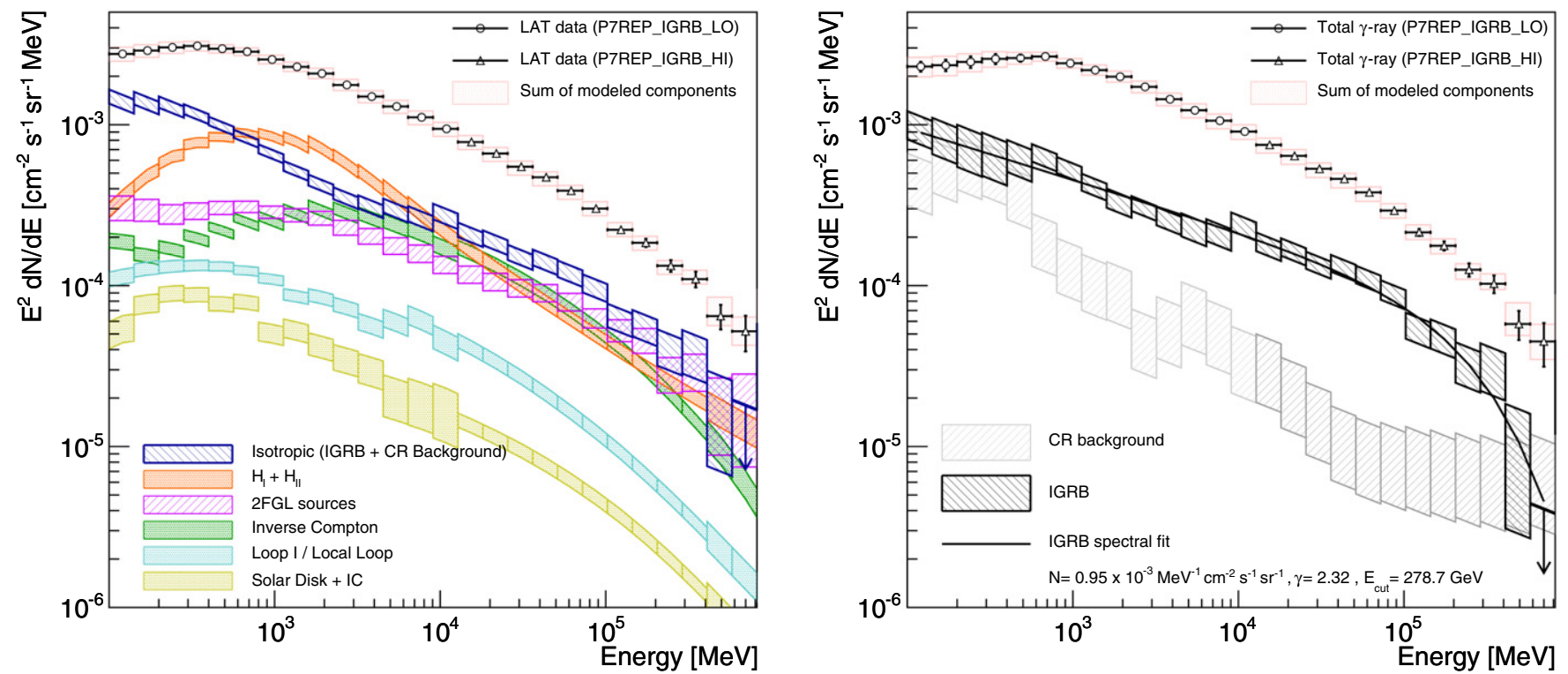

Figure 4. Results of the IGRB fit for foreground model A. Average intensities for Galactic latitudes $|b|>20^{\circ}$ are shown. Left: intensities attributed to the different foreground templates, the isotropic emission, and the individually resolved sources in the multicomponent likelihood fit. The isotropic emission and the individually resolved sources are fitted separately in each energy bin. All other components are fitted individually in each energy bin below $13 \mathrm{GeV}$ and included with fixed normalizations above this energy. The total intensity obtained from the IGRB fit is compared to the total intensity observed by the LAT. Error bars include statistical errors and systematic errors from the uncertainty in the LAT effective area parameterization. See the text for details. Right: IGRB and CR background contributions to the isotropic emission. The line indicates the best-fit IGRB spectrum with a power-law plus exponential cutoff spectral model. Spectral parameters are given in the legend. The total intensities obtained from the fit and measured by the LAT are shown with the CR background contributions subtracted in this graph.
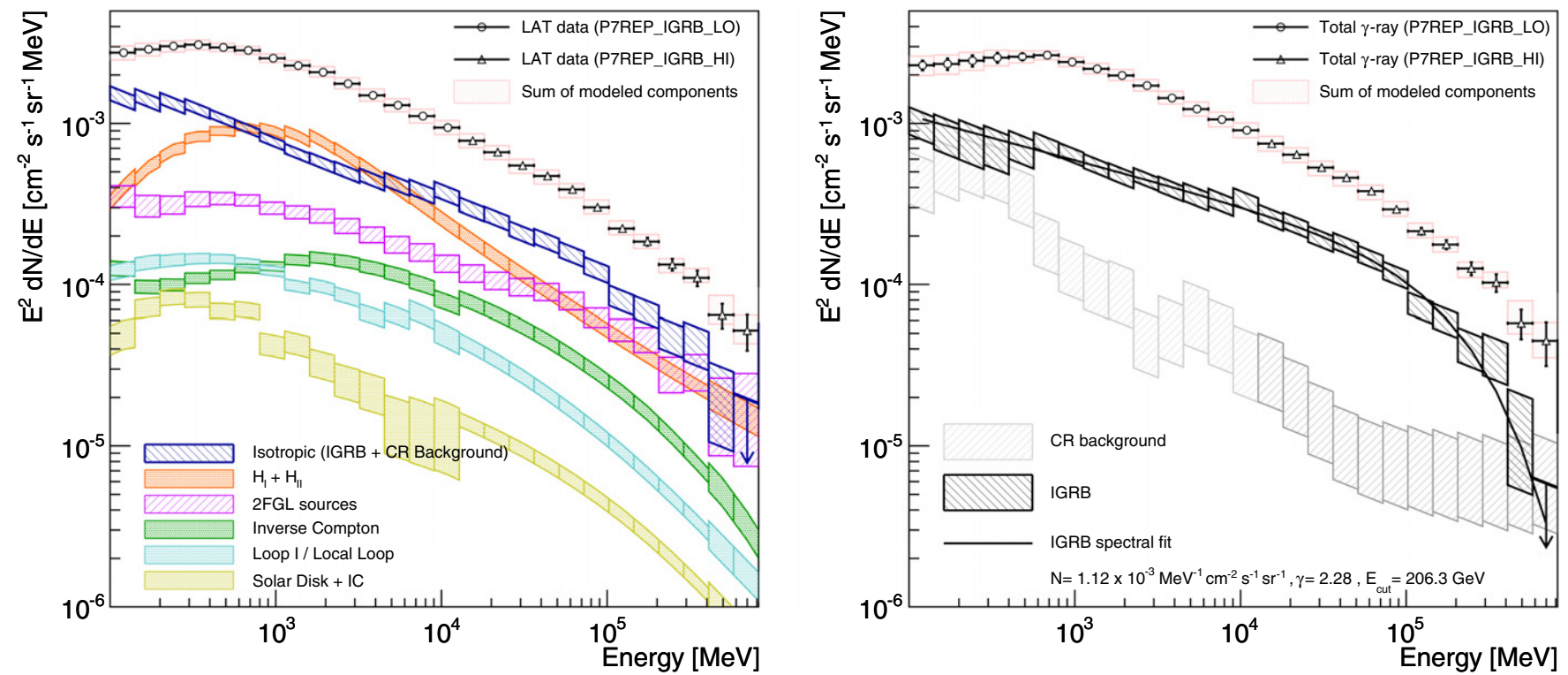

Figure 5. Results of the IGRB fit for foreground model B. See Figure 4 for legend.

CR contamination to the isotropic emission. Since the isotropic and IGRB intensities in the highest energy band are compatible with zero within the $1 \sigma$ uncertainty range, we quote upper limits in that energy band. ${ }^{78}$ The error bars displayed for the individual components in the three figures include the statistical uncertainty and the systematic uncertainty of the effective area parameterization (Ackermann et al. 2012b) added in quadrature.

${ }^{78}$ Statistical uncertainties on the isotropic emission have been calculated using the MINOS algorithm of the MINUIT minimization package (James \& Roos 1975). The position of the upper limit corresponds to the upper bound of the $1 \sigma$ uncertainty interval.
The error bars for the IGRB component additionally contain the systematic uncertainty due to subtracting a not perfectly known CR background contamination, also added in quadrature.

The IGRB intensities corresponding to foreground models A, $\mathrm{B}$, and $\mathrm{C}$ are compared in Figure 7. Numerical values for the IGRB intensities per energy band when using foreground model $A$ are presented in Table 3. Intensities for other foreground models can be found in the electronic supplementary material to this article. The IGRB intensity shows a clear cutoff at high energies, independent of the foreground model. A $\chi^{2}$ regression of the IGRB spectrum using a power law with exponential cutoff 

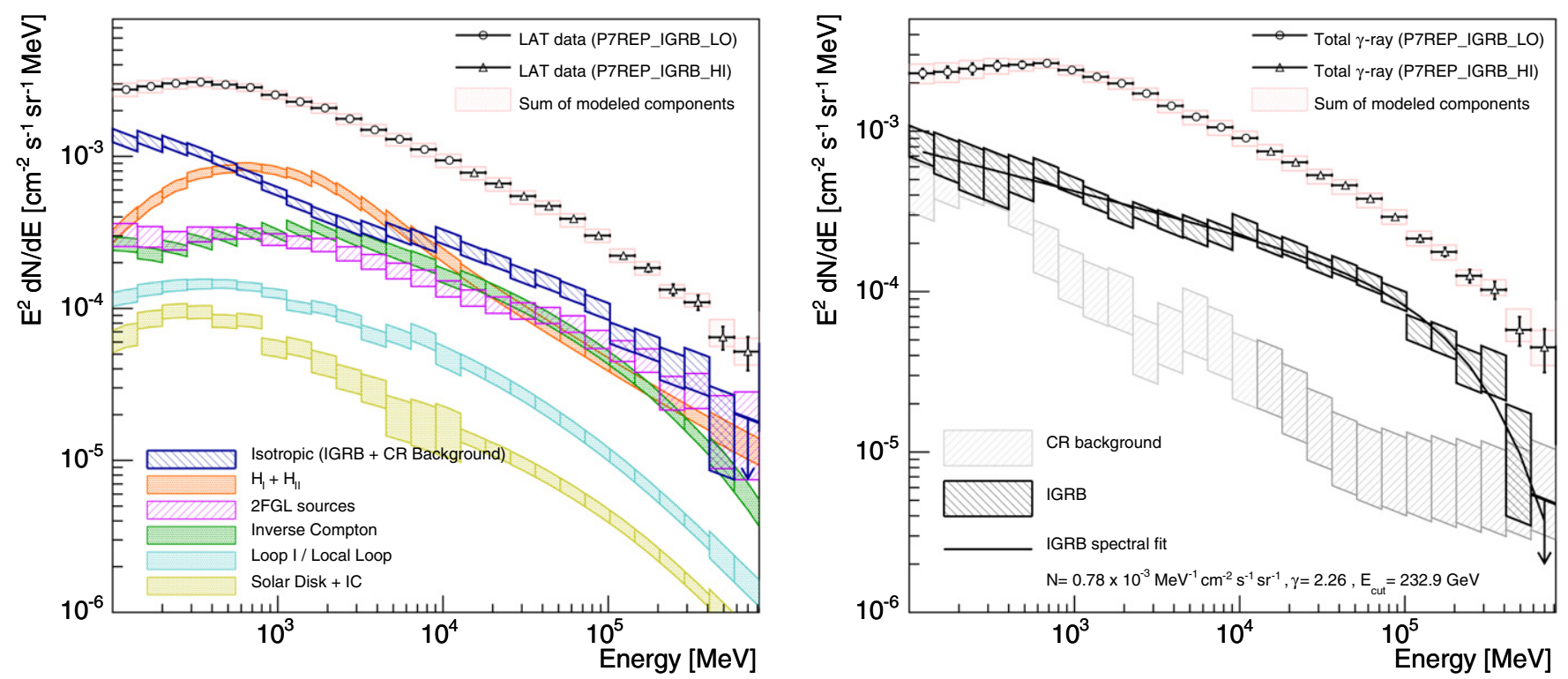

Figure 6. Results of the IGRB fit for foreground model C. See Figure 4 for legend.

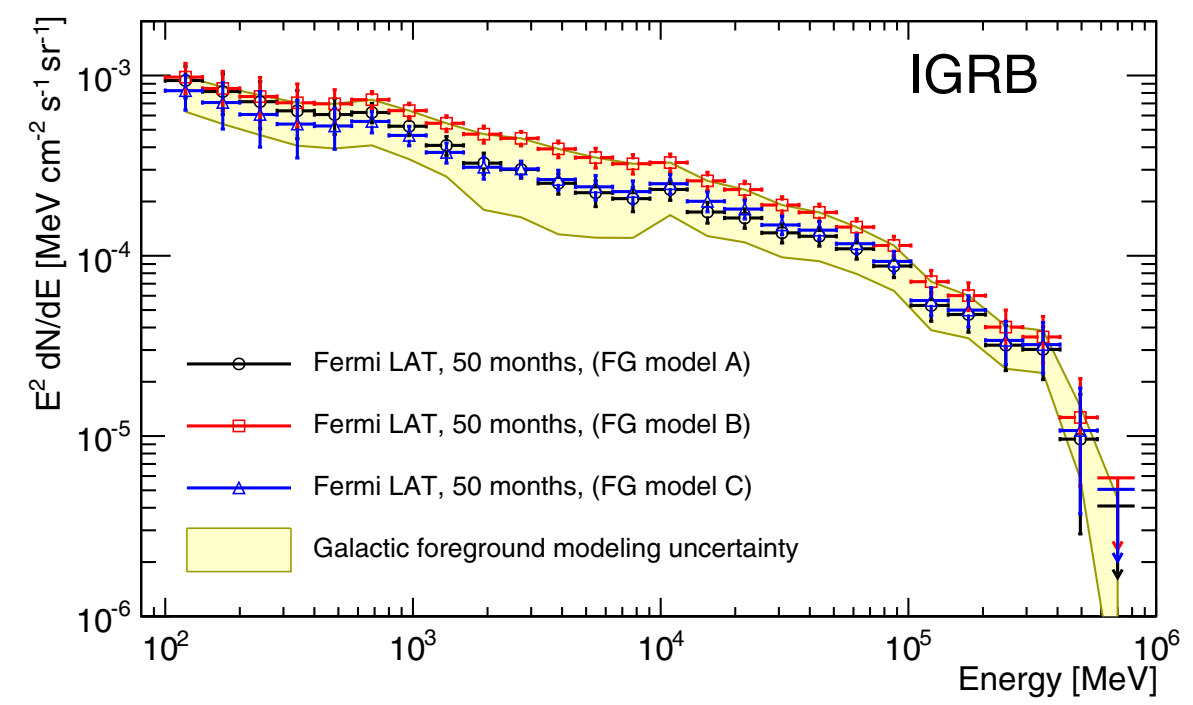

Figure 7. Comparison of the derived IGRB intensities for different foreground (FG) models. The error bars include the statistical uncertainty and systematic uncertainties from the effective area parameterization, as well as the CR background subtraction (statistical and systematic uncertainties have been added in quadrature). The shaded band indicates the systematic uncertainty arising from uncertainties in the Galactic foreground: the IGRB intensity range spanned by the three benchmark models, the variants described in Section 4.2, and the normalization uncertainties derived from the high-latitude data/model comparison. See Section 5.2 for details.

(PLE) spectral model of the form

$$
\frac{d N}{d E}=I_{100}\left(\frac{E}{100 \mathrm{MeV}}\right)^{-\gamma} \exp \left(\frac{-E}{E_{\text {cut }}}\right)
$$

results in low $\chi^{2}$ values for all three foreground models and can therefore be considered suitable to characterize the IGRB spectrum. We further try to fit the IGRB intensities with a single PL and a smoothly connected broken power law (BPL). The fit parameters for the PLE model, as well as the $\chi^{2}$ values for all fitted spectral hypotheses, are summarized in Table 4 . The $\chi^{2}$ values for the BPL and the PLE spectral models are similar enough that the two hypotheses are indistinguishable in the energy range observed. We prefer to quote fitted parameter values for the PLE model given its lower number (three versus four) of free parameters. The PL model is disfavored independent of the foreground model based on the high $\chi^{2}$ values of 88 (foreground model A), 151 (foreground model B), and 106 (foreground model C) for 23 degrees of freedom. Note that the $\chi^{2}$ value cannot be easily interpreted in terms of a significance for the agreement between spectral model and data because the error bars of the IGRB spectrum are systematics dominated over most of the energy range, and therefore correlations between bins are expected. These correlations are also responsible for the rather small $\chi^{2}$ values (when compared to the number of degrees of freedom) for the PLE and BPL spectral models.

The large difference in $\chi^{2}$ between the PL and the PLE models even when neglecting bin-to-bin correlations can still be interpreted as robust evidence against a simple PL spectrum. For the benchmark models this $\chi^{2}$ difference is larger than 61 
Table 3

IGRB and Total EGB Intensities

\begin{tabular}{|c|c|c|c|c|c|}
\hline $\begin{array}{l}\text { Energy Range } \\
(\mathrm{GeV})\end{array}$ & $\begin{array}{c}\text { IGRB } \\
\text { Intensity } \\
\left(\mathrm{cm}^{-2} \mathrm{~s}^{-1} \mathrm{sr}^{-1}\right)\end{array}$ & $\begin{array}{c}\text { FG Model Uncert. } \\
\text { on IGRB } \\
\left(\mathrm{cm}^{-2} \mathrm{~s}^{-1} \mathrm{sr}^{-1}\right)\end{array}$ & $\begin{array}{c}\text { Total EGB } \\
\text { Intensity } \\
\left(\mathrm{cm}^{-2} \mathrm{~s}^{-1} \mathrm{sr}^{-1}\right)\end{array}$ & $\begin{array}{l}\text { FG Model Uncert. } \\
\text { On Total EGB } \\
\left(\mathrm{cm}^{-2} \mathrm{~s}^{-1} \mathrm{sr}^{-1}\right)\end{array}$ & $\begin{array}{c}\text { Sources }|b|>20^{\circ} \\
\left(\mathrm{cm}^{-2} \mathrm{~s}^{-1} \mathrm{sr}^{-1}\right)\end{array}$ \\
\hline $0.10-0.14$ & $(2.8 \pm 0.6) \times 10^{-6}$ & ${ }_{-0.9}^{+0.1} \times 10^{-6}$ & $(3.7 \pm 0.6) \times 10^{-6}$ & ${ }_{-1.2}^{+0.3} \times 10^{-6}$ & $(9.0 \pm 1.6) \times 10^{-7}$ \\
\hline $0.14-0.20$ & $(1.7 \pm 0.4) \times 10^{-6}$ & ${ }_{-0.6}^{+0.1} \times 10^{-6}$ & $(2.3 \pm 0.4) \times 10^{-6}$ & ${ }_{-0.8}^{+0.1} \times 10^{-6}$ & $(6.2 \pm 1.0) \times 10^{-7}$ \\
\hline $0.20-0.28$ & $(1.1 \pm 0.3) \times 10^{-6}$ & ${ }_{-0.4}^{+0.0} \times 10^{-6}$ & $(1.5 \pm 0.3) \times 10^{-6}$ & ${ }_{-0.5}^{+0.0} \times 10^{-6}$ & $(4.1 \pm 0.6) \times 10^{-7}$ \\
\hline $0.28-0.40$ & $(6.7 \pm 2.0) \times 10^{-7}$ & ${ }_{-2.4}^{+0.7} \times 10^{-7}$ & $(9.7 \pm 2.0) \times 10^{-7}$ & ${ }_{-3.2}^{+1.2} \times 10^{-7}$ & $(3.0 \pm 0.4) \times 10^{-7}$ \\
\hline $0.40-0.57$ & $(4.5 \pm 1.0) \times 10^{-7}$ & ${ }_{-1.6}^{+0.7} \times 10^{-7}$ & $(6.7 \pm 1.0) \times 10^{-7}$ & ${ }_{-2.2}^{+0.9} \times 10^{-7}$ & $(2.2 \pm 0.2) \times 10^{-7}$ \\
\hline $0.57-0.80$ & $(3.3 \pm 0.4) \times 10^{-7}$ & ${ }_{-1.1}^{+0.6} \times 10^{-7}$ & $(4.9 \pm 0.4) \times 10^{-7}$ & ${ }_{-1.6}^{+0.7} \times 10^{-7}$ & $(1.6 \pm 0.1) \times 10^{-7}$ \\
\hline $0.80-1.1$ & $(1.9 \pm 0.2) \times 10^{-7}$ & ${ }_{-0.7}^{+0.4} \times 10^{-7}$ & $(3.0 \pm 0.2) \times 10^{-7}$ & ${ }_{-1.0}^{+0.5} \times 10^{-7}$ & $(1.1 \pm 0.1) \times 10^{-7}$ \\
\hline $1.1-1.6$ & $(1.1 \pm 0.1) \times 10^{-7}$ & ${ }_{-0.4}^{+0.3} \times 10^{-7}$ & $(1.8 \pm 0.1) \times 10^{-7}$ & ${ }_{-0.6}^{+0.4} \times 10^{-7}$ & $(7.1 \pm 0.7) \times 10^{-8}$ \\
\hline $1.6-2.3$ & $(6.0 \pm 0.8) \times 10^{-8}$ & $\pm 2.7 \times 10^{-8}$ & $(1.1 \pm 0.1) \times 10^{-7}$ & $\pm 0.3 \times 10^{-7}$ & $(4.8 \pm 0.5) \times 10^{-8}$ \\
\hline $2.3-3.2$ & $(3.9 \pm 0.4) \times 10^{-8}$ & ${ }_{-1.8}^{+1.9} \times 10^{-8}$ & $(6.9 \pm 0.5) \times 10^{-8}$ & ${ }_{-2.1}^{+1.9} \times 10^{-8}$ & $(3.0 \pm 0.3) \times 10^{-8}$ \\
\hline $3.2-4.5$ & $(2.3 \pm 0.3) \times 10^{-8}$ & ${ }_{-1.1}^{+1.3} \times 10^{-8}$ & $(4.2 \pm 0.4) \times 10^{-8}$ & ${ }_{-1.2}^{+1.3} \times 10^{-8}$ & $(1.9 \pm 0.2) \times 10^{-8}$ \\
\hline $4.5-6.4$ & $(1.5 \pm 0.2) \times 10^{-8}$ & ${ }_{-0.6}^{+0.8} \times 10^{-8}$ & $(2.6 \pm 0.3) \times 10^{-8}$ & ${ }_{-0.7}^{+0.8} \times 10^{-8}$ & $(1.1 \pm 0.1) \times 10^{-8}$ \\
\hline $6.4-9.1$ & $(9.6 \pm 1.5) \times 10^{-9}$ & $\begin{array}{l}+5.4 \\
-3.8\end{array} \times 10^{-9}$ & $(1.7 \pm 0.2) \times 10^{-8}$ & $\pm 0.5 \times 10^{-8}$ & $(7.3 \pm 0.9) \times 10^{-9}$ \\
\hline $9.1-13$ & $(7.6 \pm 1.0) \times 10^{-9}$ & ${ }_{-2.1}^{+3.1} \times 10^{-9}$ & $(1.2 \pm 0.1) \times 10^{-8}$ & $\pm 0.3 \times 10^{-8}$ & $(4.4 \pm 0.5) \times 10^{-9}$ \\
\hline $13-18$ & $(4.0 \pm 0.5) \times 10^{-9}$ & $+2.0 \times 10^{-9}$ & $(6.8 \pm 0.6) \times 10^{-9}$ & ${ }_{-1.9}^{+2.0} \times 10^{-9}$ & $(2.7 \pm 0.3) \times 10^{-9}$ \\
\hline $18-26$ & $(2.6 \pm 0.3) \times 10^{-9}$ & ${ }_{-0.7}^{+1.2} \times 10^{-9}$ & $(4.4 \pm 0.4) \times 10^{-9}$ & $\pm 1.2 \times 10^{-9}$ & $(1.7 \pm 0.2) \times 10^{-9}$ \\
\hline $26-36$ & $(1.6 \pm 0.2) \times 10^{-9}$ & ${ }_{-0.4}^{+0.7} \times 10^{-9}$ & $(2.7 \pm 0.2) \times 10^{-9}$ & $\pm 0.7 \times 10^{-9}$ & $(1.1 \pm 0.1) \times 10^{-9}$ \\
\hline $36-51$ & $(1.1 \pm 0.1) \times 10^{-9}$ & ${ }_{-0.3}^{+0.4} \times 10^{-9}$ & $(1.8 \pm 0.2) \times 10^{-9}$ & ${ }_{-0.5}^{+0.4} \times 10^{-9}$ & $(7.3 \pm 0.9) \times 10^{-10}$ \\
\hline $51-72$ & $(6.3 \pm 0.8) \times 10^{-10}$ & ${ }_{-1.7}^{+2.0} \times 10^{-10}$ & $(1.1 \pm 0.1) \times 10^{-9}$ & ${ }_{-0.3}^{+0.2} \times 10^{-9}$ & $(4.5 \pm 0.6) \times 10^{-10}$ \\
\hline $72-100$ & $(3.6 \pm 0.5) \times 10^{-10}$ & ${ }_{-1.0}^{+1.1} \times 10^{-10}$ & $(6.2 \pm 0.6) \times 10^{-10}$ & ${ }_{-1.7}^{+1.1} \times 10^{-10}$ & $(2.6 \pm 0.3) \times 10^{-10}$ \\
\hline $100-140$ & $(1.5 \pm 0.3) \times 10^{-10}$ & ${ }_{-0.4}^{+0.5} \times 10^{-10}$ & $(3.1 \pm 0.4) \times 10^{-10}$ & ${ }_{-0.9}^{+0.5} \times 10^{-10}$ & $(1.5 \pm 0.2) \times 10^{-10}$ \\
\hline $140-200$ & $(9.8 \pm 2.0) \times 10^{-11}$ & ${ }_{-2.6}^{+2.7} \times 10^{-11}$ & $(1.9 \pm 0.3) \times 10^{-10}$ & ${ }_{-0.5}^{+0.3} \times 10^{-10}$ & $(9.3 \pm 1.6) \times 10^{-11}$ \\
\hline $200-290$ & $\left(4.7_{-1.3}^{+1.4}\right) \times 10^{-11}$ & ${ }_{-1.2}^{+1.3} \times 10^{-11}$ & $(8.9 \pm 1.7) \times 10^{-11}$ & ${ }_{-2.4}^{+1.3} \times 10^{-11}$ & $(4.1 \pm 1.0) \times 10^{-11}$ \\
\hline $290-410$ & $\left(3.2_{-1.0}^{+1.1}\right) \times 10^{-11}$ & ${ }_{-0.8}^{+0.9} \times 10^{-11}$ & $(6.3 \pm 1.3) \times 10^{-11}$ & ${ }_{-1.7}^{+0.9} \times 10^{-11}$ & $(3.0 \pm 0.8) \times 10^{-11}$ \\
\hline $410-580$ & $\left(7.3_{-5.1}^{+5.7}\right) \times 10^{-12}$ & ${ }_{-2.9}^{+3.8} \times 10^{-12}$ & $\left(2.1_{-0.8}^{+0.9}\right) \times 10^{-11}$ & ${ }_{-0.5}^{+0.4} \times 10^{-11}$ & $(1.3 \pm 0.6) \times 10^{-11}$ \\
\hline $580-820$ & $<2.3 \times 10^{-12}$ & & $(9.7 \pm 6.0) \times 10^{-12}$ & ${ }_{-2.8}^{+2.3} \times 10^{-12}$ & $(9.0 \pm 5.2) \times 10^{-12}$ \\
\hline
\end{tabular}

Notes. Measured intensities of the IGRB, the total EGB, and the identified sources $\left(|b|>20^{\circ}\right)$ per energy band, when using model A to describe the Galactic foreground. Uncertainties arising from foreground (FG) modeling are given in separate columns. Digitized versions of this table and the corresponding results for foreground models $\mathrm{B}$ and $\mathrm{C}$ are available in the online supplementary materials.

(This table is available in its entirety in machine-readable form.)

Table 4

Results of the Parametric Fit of the IGRB

\begin{tabular}{|c|c|c|c|c|c|c|c|}
\hline $\begin{array}{l}\text { Foreground } \\
\text { Model }\end{array}$ & $\begin{array}{c}I_{100} \\
\left(\mathrm{MeV}^{-1} \mathrm{~cm}^{-2} \mathrm{~s}^{-1} \mathrm{sr}^{-1}\right)\end{array}$ & $\gamma$ & $\begin{array}{c}E_{\text {cut }} \\
(\mathrm{GeV})\end{array}$ & $\begin{array}{c}I_{>100} \\
\left(\mathrm{~cm}^{-2} \mathrm{~s}^{-1} \mathrm{sr}^{-1}\right)\end{array}$ & $\begin{array}{c}\chi^{2} / \text { ndof } \\
\left(\mathrm{PLE}^{\mathrm{a}}\right)\end{array}$ & $\begin{array}{l}\chi^{2} / \text { ndof } \\
\left(\mathrm{PL}^{\mathrm{a}}\right)\end{array}$ & $\begin{array}{l}\chi^{2} / \mathrm{ndof}^{2} \\
\left(\mathrm{BPL}^{\mathrm{a}}\right)\end{array}$ \\
\hline Model A & $(0.95 \pm 0.08) \times 10^{-7}$ & $2.32 \pm 0.02$ & $279 \pm 52$ & $(7.2 \pm 0.6) \times 10^{-6}$ & $13.9 / 23$ & $87.5 / 24$ & $13.5 / 22$ \\
\hline Model B & $(1.12 \pm 0.08) \times 10^{-7}$ & $2.28 \pm 0.02$ & $206 \pm 31$ & $(8.7 \pm 0.6) \times 10^{-6}$ & $7.9 / 23$ & $151 . / 24$ & $10.6 / 22$ \\
\hline
\end{tabular}

Notes. Parameters obtained from a parametric fit of the IGRB spectrum. Intensity $I_{100}$, spectral index $\gamma$, and cutoff energy $E_{\text {cut }}$ for a fit of the observed spectrum with the function given in Equation (1) are shown in Columns 2-4. The integrated IGRB intensity above $100 \mathrm{MeV}, I_{>100}$, is found in Column 5. A comparison of the $\chi^{2} /$ ndof values between the fit with the function in Equation (1) and alternative spectral models is given in Columns 6-8. The $\chi^{2}$ values include systematic uncertainty. ${ }^{\mathrm{a}} \mathrm{PLE}=$ power-law plus exponential cutoff; $\mathrm{PL}=$ power law; $\mathrm{BPL}=$ broken power law.

at just one added degree of freedom in the model. We also calculated the $\chi^{2}$ differences between the PL and PLE models for the additional foreground models used in the investigation of the foreground-related systematics that are summarized in Table 4. The $\chi^{2}$ difference between the PL and PLE models is 45 or larger in all of these foreground variations.

Finally, we calculated the $\chi^{2}$ difference between PL and PLE models if we add the foreground-model-related systematic uncertainties to the instrument-related uncertainties. Since we do not know the correlations between the bins introduced by the systematic errors, we adopt a worst-case scenario here assuming that the dominant fraction of the systematic error is fully correlated at low energies and anticorrelated between low and high energies $(E>300 \mathrm{GeV})$. Such a hypothetical anticorrelation in the systematics might artificially enhance indications of a cutoff. Even in this worst-case scenario we still find $\chi^{2}$ differences exceeding 25 between the PL and PLE scenarios for our three benchmark models.

Residual maps of the relative deviations in intensity between model and data in different regions of the sky can be found 
Table 5

Impact of Foreground Model Variations on IGRB Spectral Parameters

\begin{tabular}{|c|c|c|c|c|}
\hline Model Variation & $\begin{array}{c}I_{100} \\
\left(\mathrm{MeV}^{-1} \mathrm{~cm}^{-2} \mathrm{~s}^{-1} \mathrm{sr}^{-1}\right)\end{array}$ & $\gamma$ & $\begin{array}{c}E_{\text {cut }} \\
(\mathrm{GeV})\end{array}$ & $\chi^{2} /$ ndof \\
\hline $10 \times$ ISRF in Galactic bulge & $(0.96 \pm 0.08) \times 10^{-7}$ & $2.31 \pm 0.02$ & $273 \pm 50$ & $13.4 / 23$ \\
\hline Random magnetic field strength $3 \mu \mathrm{G}$ & $(0.93 \pm 0.09) \times 10^{-7}$ & $2.33 \pm 0.03$ & $257 \pm 56$ & $14.1 / 23$ \\
\hline Plain diffusion model & $(0.91 \pm 0.08) \times 10^{-7}$ & $2.31 \pm 0.02$ & $264 \pm 52$ & $13.4 / 23$ \\
\hline Model $38^{\mathrm{a}}$ (Pulsars trace CR sources, $4 \mathrm{kpc}$ CR halo) & $(1.06 \pm 0.09) \times 10^{-7}$ & $2.33 \pm 0.02$ & $367 \pm 75$ & $15.0 / 23$ \\
\hline Model $62^{\mathrm{a}}$ (Pulsars trace CR sources, $10 \mathrm{kpc}$ CR halo) & $(0.89 \pm 0.08) \times 10^{-7}$ & $2.31 \pm 0.02$ & $374 \pm 77$ & $16.7 / 23$ \\
\hline Fermi bubbles template A & $(1.02 \pm 0.09) \times 10^{-7}$ & $2.32 \pm 0.02$ & $229 \pm 44$ & $13.0 / 23$ \\
\hline Fermi bubbles template B & $(1.05 \pm 0.09) \times 10^{-7}$ & $2.31 \pm 0.02$ & $244 \pm 42$ & $13.7 / 23$ \\
\hline H I-to-dust ratio $+10 \%$ & $(1.09 \pm 0.09) \times 10^{-7}$ & $2.34 \pm 0.02$ & $280 \pm 52$ & $12.0 / 23$ \\
\hline H I-to-dust ratio $-10 \%$ & $(0.82 \pm 0.08) \times 10^{-7}$ & $2.30 \pm 0.03$ & $274 \pm 51$ & $17.5 / 23$ \\
\hline
\end{tabular}

Notes. Parameters obtained from fits of the IGRB spectrum for variants of the DGE foreground model. Specific intensity $I_{100}$, spectral index $\gamma$, and cutoff energy $E_{\text {cut }}$ for a fit of the observed spectrum with the function given in Equation (1) are shown in Columns $2-4$. The $\chi^{2} /$ ndof values of the fit are shown in Column 5.

a From Ackermann et al. (2012g).

${ }^{b}$ Model is not well fitted by a simple power-law with exponential cutoff spectral hypothesis. A two-component fit is used instead for this model for the evaluation of the foreground model systematics. The parameters obtained from this fit are $I_{100}^{(0)}=0.69 \times 10^{-7} \mathrm{GeV}^{-1} \mathrm{~cm}^{-2} \mathrm{~s}^{-1} \mathrm{sr}^{-1}, \gamma^{(0)}=1.74$, and $E_{\mathrm{cut}}^{(0)}=0.60 \mathrm{GeV}$ for the first component, and $I_{100}^{(1)}=0.16 \times 10^{-7} \mathrm{GeV}^{-1} \mathrm{~cm}^{-2} \mathrm{~s}^{-1} \mathrm{sr}^{-1}, \gamma^{(1)}=2.02$, and $E_{\mathrm{cut}}^{(1)}=183 \mathrm{GeV}$ for the second component.

in Appendix B. None of the models considered are a perfect description of the data, and large-scale residuals at the $25 \%$ level appear in many parts of the sky. No Galactic foreground model is unambiguously preferred over the others based on the level and distribution of the residual $\gamma$-ray emission. Prominent residuals include the Fermi bubbles, but other unmodeled residuals appear in different parts of the sky. Also, there seems to be less intensity observed in the south polar region than in its northern counterpart, a feature that cannot be modeled with the classes of foreground models considered here, which exhibit a symmetrical CR density about the Galactic plane by construction.

In the next section we present a study of the general impact of such unmodeled residuals on the IGRB spectrum. The study is not restricted to the Fermi bubbles, but applies to all features in the residual maps.

\subsection{Systematic Uncertainties from Foreground Modeling}

The fitting procedure is applied to the variants of the foreground models introduced in Section 4.2 in the same way as for the benchmark models A, B, and C. Table 5 summarizes the spectral parameter and $\chi^{2}$ values obtained for the IGRB when using each of the foreground model variants. For most of the variant models, the general shape of the IGRB spectrum is not affected; a simple PLE remains a valid parameterization. The single exception is the effect from changing the source distribution. When using the distribution of SNRs as a tracer of the CR source density, a second apparent spectral feature manifests as a dip in the IGRB spectrum at a few GeV. This dip in the IGRB compensates the higher intensity of the IC emission with respect to other foreground models. A good fit of the shape of the IGRB spectrum can be obtained only by describing it as a sum of two components. We choose two components each having a PLE spectrum to describe the resulting IGRB spectrum for this foreground model version.

A second investigation of systematic uncertainties is aimed at the residuals visible in the maps shown in Appendix B. We study the variations of differences between LAT data and our model within four very high latitude $\left(|b|>60^{\circ}\right)$ overlapping regions in which the IGRB is responsible for a substantial fraction of the total $\gamma$-ray emission. We use the Galactic north pole, the Galactic south pole, the $|b|>60^{\circ}$ region facing the inner Galaxy, and the $|b|>60^{\circ}$ region facing the outer Galaxy. For each of these regions, we calculate the spectral residual and the renormalization factor for the IGRB needed to obtain the best overall agreement between model and data in the corresponding region. The resulting adjustment factors are between 0.7 and 0.95 depending on the region and foreground model. Note that all adjustment factors are $<1$, i.e., the observed $\gamma$-ray intensity at $|b|>60^{\circ}$ is lower than that predicted by the models. This overestimation of the $\gamma$-ray intensity could arise either from an overestimation of the Galactic foreground at high latitudes or from an overestimation of the isotropic intensity and therefore represents a systematic uncertainty for our analysis.

Including this second study, we find that the normalization of the IGRB intensity $I_{>100}$ varies by $+15 \% /-30 \%$ with respect to foreground model A depending on our assumptions about the DGE foreground, while the spectral index varies between 2.26 and 2.34, and the cutoff energy between 206 and $374 \mathrm{GeV}$. Summarizing the results above, the systematic uncertainty in the IGRB spectrum associated with foreground modeling is shown in Figure 7.

\subsection{Total EGB Intensity}

The total EGB as defined in Section 1 is a quantity independent of the instrument and observation time. For the purpose of this analysis, we call the sum of the IGRB and the sky-averaged intensity of high-latitude $|b|>20^{\circ}$ resolved LAT sources the total EGB. We note that this definition of the total EGB formally includes a small fraction of Galactic sources, e.g., millisecond pulsars. However, the contributions of known high-latitude pulsars extracted from the second LAT pulsar catalog (Abdo et al. 2013) are less than 5\% of the total EGB anywhere in the measured energy range, well below the level of systematic uncertainty inherent to the IGRB measurement. We further check the variation of the total intensity when varying the latitude threshold from $|b|>20^{\circ}$ to $|b|>40^{\circ}$ to probe a possible Galactic source contamination. We find that the derived intensities are consistent within the respective uncertainties for each latitude threshold. 


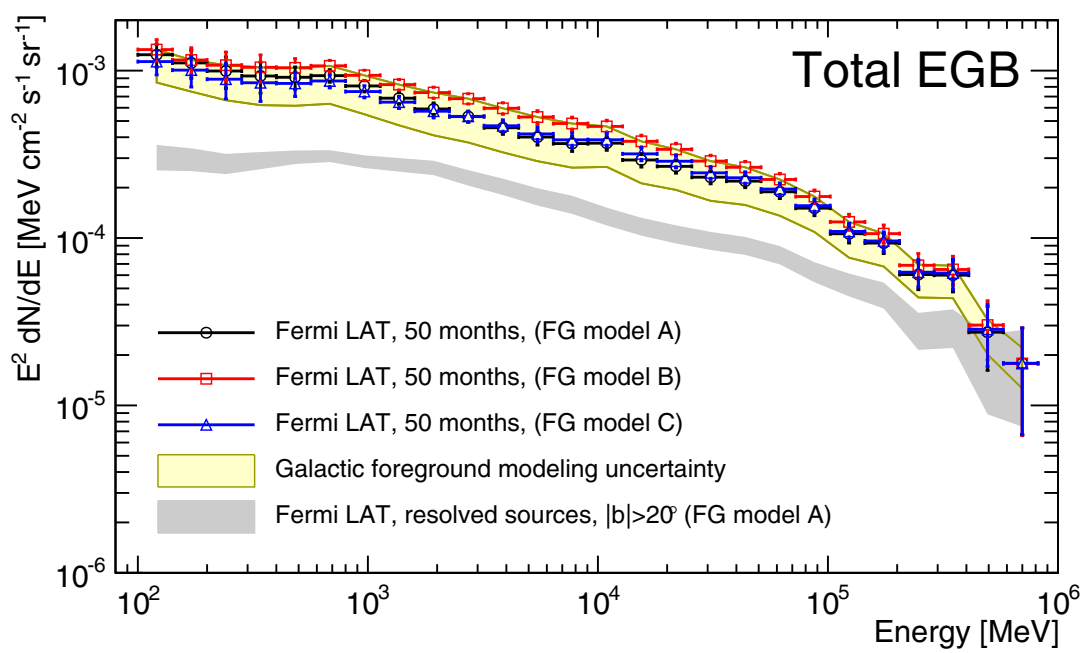

Figure 8. Comparison of the total EGB intensities for different foreground models. The total EGB intensity is obtained by summing the IGRB intensity and the cumulative intensity from resolved Fermi LAT sources at latitudes $|b|>20^{\circ}$ (gray band). See Figure 7 for legend.

Table 6

Results of the Parametric Fit of the Total EGB

\begin{tabular}{|c|c|c|c|c|c|c|c|}
\hline $\begin{array}{l}\text { Foreground } \\
\text { Model }\end{array}$ & $\begin{array}{c}I_{100} \\
\left(\mathrm{MeV}^{-1} \mathrm{~cm}^{-2} \mathrm{~s}^{-1} \mathrm{sr}^{-1}\right)\end{array}$ & $\gamma$ & $\begin{array}{c}E_{\text {cut }} \\
(\mathrm{GeV})\end{array}$ & $\begin{array}{c}I_{>100} \\
\left(\mathrm{~cm}^{-2} \mathrm{~s}^{-1} \mathrm{sr}^{-1}\right)\end{array}$ & $\begin{array}{l}\chi^{2} / \text { ndof } \\
\left(\mathrm{PLE}^{\mathrm{a}}\right)\end{array}$ & $\begin{array}{l}\chi^{2} / \text { ndof } \\
\left(\mathrm{PL}^{\mathrm{a}}\right)\end{array}$ & $\begin{array}{l}\chi^{2} / \text { ndof } \\
\left(\mathrm{BPL}^{\mathrm{a}}\right)\end{array}$ \\
\hline Model A & $(1.48 \pm 0.09) \times 10^{-7}$ & $2.31 \pm 0.02$ & $362 \pm 64$ & $(1.13 \pm 0.07) \times 10^{-5}$ & $11.0 / 23$ & $72.4 / 24$ & $10.5 / 22$ \\
\hline Model B & $(1.66 \pm 0.09) \times 10^{-7}$ & $2.28 \pm 0.01$ & $267 \pm 37$ & $(1.29 \pm 0.07) \times 10^{-5}$ & $13.5 / 23$ & $130 . / 24$ & $11.3 / 22$ \\
\hline Model C & $(1.28 \pm 0.08) \times 10^{-7}$ & $2.30 \pm 0.02$ & $366 \pm 71$ & $(0.98 \pm 0.06) \times 10^{-5}$ & $6.9 / 23$ & $91.1 / 24$ & $7.7 / 22$ \\
\hline
\end{tabular}

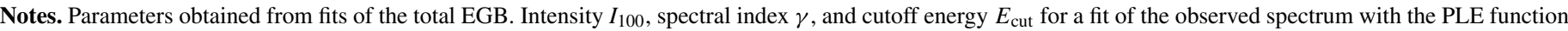

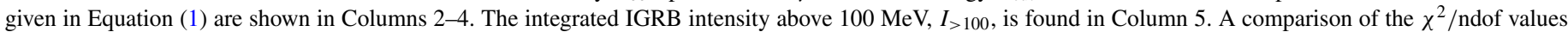
between the fit with the function in Equation (1) and alternative spectral models is given in Columns 6-8. The $\chi^{2}$ values include systematic uncertainties.

${ }^{a} \mathrm{PLE}=$ power-law plus exponential cutoff; $\mathrm{PL}=$ power law; $\mathrm{BPL}=$ broken power law .

Figure 8 compares the total EGB derived for foreground models A, B, and C, respectively. Numerical values for the total EGB intensities per energy band are given in Table 3. Again, we use a $\chi^{2}$ regression to test different functional parameterizations of the spectrum. The best-fit parameters for a fit with a PLE and $\chi^{2}$ values for all tested spectral models are summarized in Table 6. For the total EGB we find similarly as for the IGRB that a PLE describes the spectral shape significantly better than an unbroken PL. The cutoff energy is higher for the total EGB than for the IGRB. As in the case of the IGRB, we cannot distinguish an exponential cutoff spectral model from a BPL. Results of the spectral fits are summarized in Table 6.

Systematic uncertainties in the total EGB spectrum arising from modeling the Galactic foreground are indicated by the shaded band in Figure 8, constructed using the identical methods described in Section 5.2 for the IGRB.

\section{DISCUSSION AND CONCLUSIONS}

We have refined the measurement of the LAT IGRB intensity relative to the analysis of Abdo et al. (2010b), which was based on 10 months of LAT observations, now using 50 months of accumulated data. The measurement lower bound has been extended from 200 to $100 \mathrm{MeV}$, and we report the first IGRB measurement with any instrument between 102 and $820 \mathrm{GeV}$.

The updated LAT IGRB spectrum remains consistent with a featureless PL between $100 \mathrm{MeV}$ and $100 \mathrm{GeV}$, and there is now strong evidence for a high-energy cutoff feature. The spectrum is well described by a PLE over the full analyzed energy range from $100 \mathrm{MeV}$ to $820 \mathrm{GeV}$. For each of the three benchmark DGE models considered here, the PL index of the IGRB is $\approx 2.3$ and the cutoff energy is $\approx 250 \mathrm{GeV}$ (Table 4 ).

The total EGB is derived by adding resolved high-latitude LAT sources (taken to be primarily extragalactic) to the measured IGRB intensity. At an energy of $100 \mathrm{GeV}$, roughly half of the total EGB intensity has now been resolved into individual sources by the LAT, predominantly blazars of the BL Lacertae type. (The demographics of LAT sources detected at energies above $10 \mathrm{GeV}$ are discussed in Ackermann et al. 2013c). The relative contribution of resolved sources becomes even more pronounced at energies exceeding $100 \mathrm{GeV}$.

The intensities of the IGRB and the total EGB are compared to the first LAT measurement of the IGRB in Abdo et al. (2010b) in Figure 9. The two are compatible within the respective systematic uncertainties. Differences can be attributed to the combined effects of a more accurate estimate of the CR background at low energies and changes in the Galactic foreground model. Importantly, the model for atmospheric secondaries has been refined to address discrepancies between data and simulation. The revised background rate of misclassified CRs is up to $50 \%$ higher at a few hundred $\mathrm{MeV}$ than the older estimates. This change contributes to a reduced integrated IGRB intensity above $100 \mathrm{MeV}$ of $7.2 \pm 0.6 \times 10^{-6}$ photons $\mathrm{cm}^{-2} \mathrm{~s}^{-1} \mathrm{sr}^{-1}$ in comparison to the $1.03 \pm 0.17 \times 10^{-5}$ photons $\mathrm{cm}^{-2} \mathrm{~s}^{-1} \mathrm{sr}^{-1}$ reported in Abdo et al. (2010b).

The intensity resolved into individual sources at latitudes $|b|>20^{\circ}$ did not change substantially between the two measurements. This is consistent with the findings in Abdo 


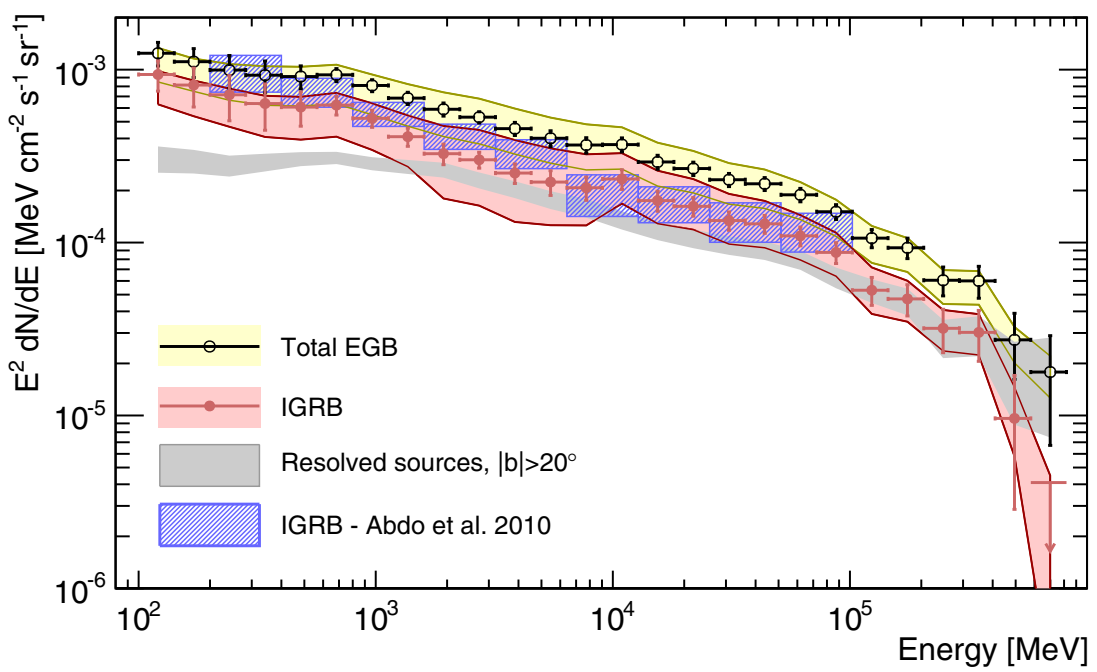

Figure 9. Comparison of the measured IGRB and total EGB intensities (foreground model A) to the first measurement of the IGRB in Abdo et al. (2010b) based on 10 months of LAT data. The error bars on the LAT measurements include the statistical uncertainty and systematic uncertainties from the effective area parameterization, as well as the CR background subtraction. Statistical and systematic uncertainties have been added in quadrature. The shaded bands indicate the systematic uncertainty arising from uncertainties in the Galactic foreground. The total EGB intensity is the sum of the IGRB and the intensity of the resolved LAT sources at high Galactic latitudes, $|b|>20^{\circ}$.

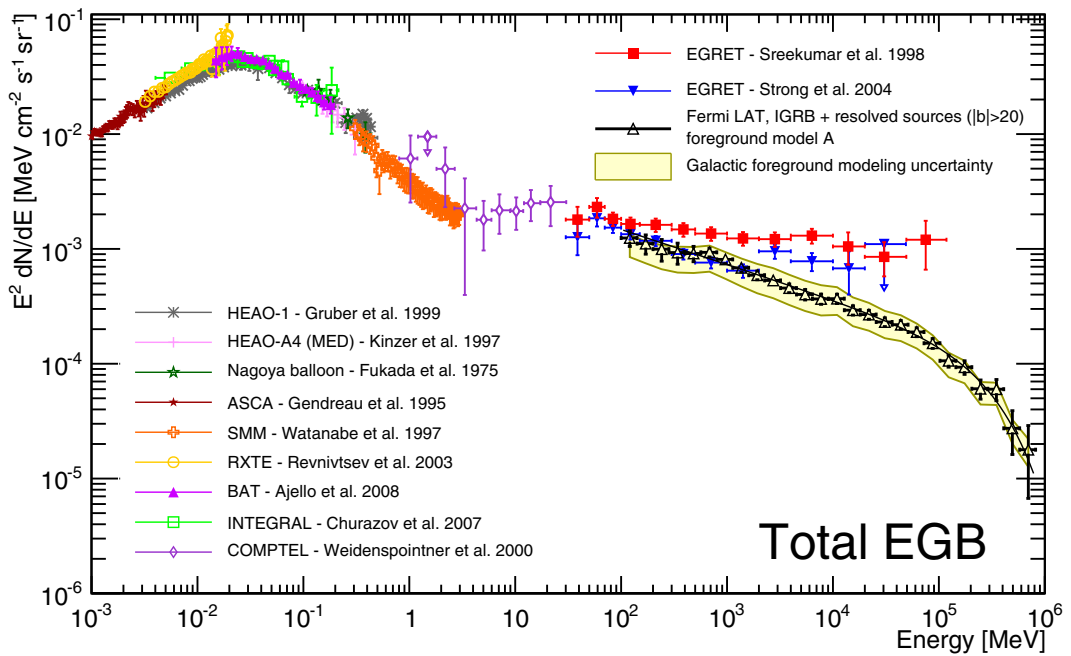

Figure 10. Comparison of the derived total EGB intensity (foreground model A) with other measurements of the X-ray and $\gamma$-ray background. The error bars on the LAT measurement include the statistical uncertainty and systematic uncertainties from the effective area parameterization, as well as the CR background subtraction. Statistical and systematic uncertainties have been added in quadrature. The shaded band indicates the systematic uncertainty arising from uncertainties in the Galactic foreground. (Note that the EGRET measurements shown are measurements of the IGRB. However, EGRET was more than an order of magnitude less sensitive to resolve individual sources on the sky than the Fermi LAT.)

et al. (2010c) and Ackermann et al. (2011a) that report a skyaveraged intensity of sources from Galactic latitudes $|b|>10^{\circ}$ of $4.0 \times 10^{-6}$ photons $\mathrm{cm}^{-2} \mathrm{~s}^{-1} \mathrm{sr}^{-1}$ after one year and $4.4 \times 10^{-6}$ photons $\mathrm{cm}^{-2} \mathrm{~s}^{-1} \mathrm{sr}^{-1}$ after two years of observations above $100 \mathrm{MeV}$. This difference corresponds to only $\approx 5 \%$ of the IGRB intensity.

Figure 10 places LAT measurements of the total EGB intensity in context with other measurements of the extragalactic $\mathrm{X}$-ray and $\gamma$-ray backgrounds, together spanning nearly nine orders of magnitude in energy between $1 \mathrm{keV}$ and $820 \mathrm{GeV}$. There is a good agreement between the total EGB measured by the LAT and the previous measurement of the IGRB using EGRET data (Sreekumar et al. 1998; Strong et al. 2004) below $1 \mathrm{GeV}$. The IGRB measured by the LAT is lower than the
EGRET IGRB measurement, as expected from the greatly superior sensitivity of the LAT to resolve individual sources when compared to EGRET.

As discussed in Section 1, numerous source populations and truly diffuse processes are expected to contribute to the EGB intensity. A detailed review of the expected contributions of specific source populations and diffuse processes is beyond the scope of this work. Instead, we focus on general constraints that can be applied to extragalactic $\gamma$-ray source populations based on the EGB spectrum, taking into account the effects of EBL attenuation. Other efforts to statistically characterize the EGB properties considering the fluctuations of counts in spatial pixels (Malyshev \& Hogg 2011) and two-point correlation functions (Ackermann et al. 2012a) have proven valuable for 

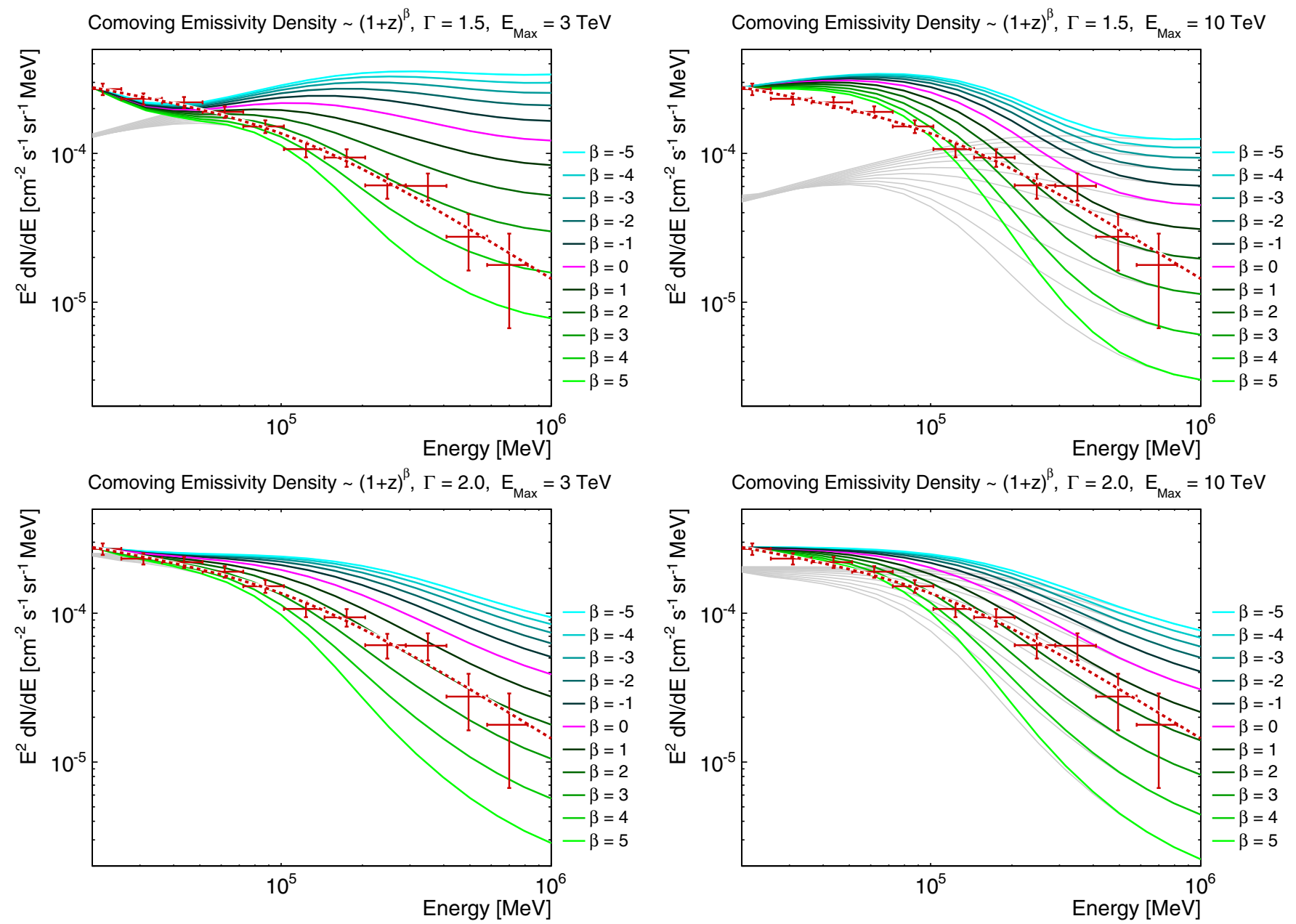

Comoving Emissivity Density $\sim(1+\mathrm{z})^{\beta}, \Gamma=2.3, \mathrm{E}_{\mathrm{Max}}=3 \mathrm{TeV}$
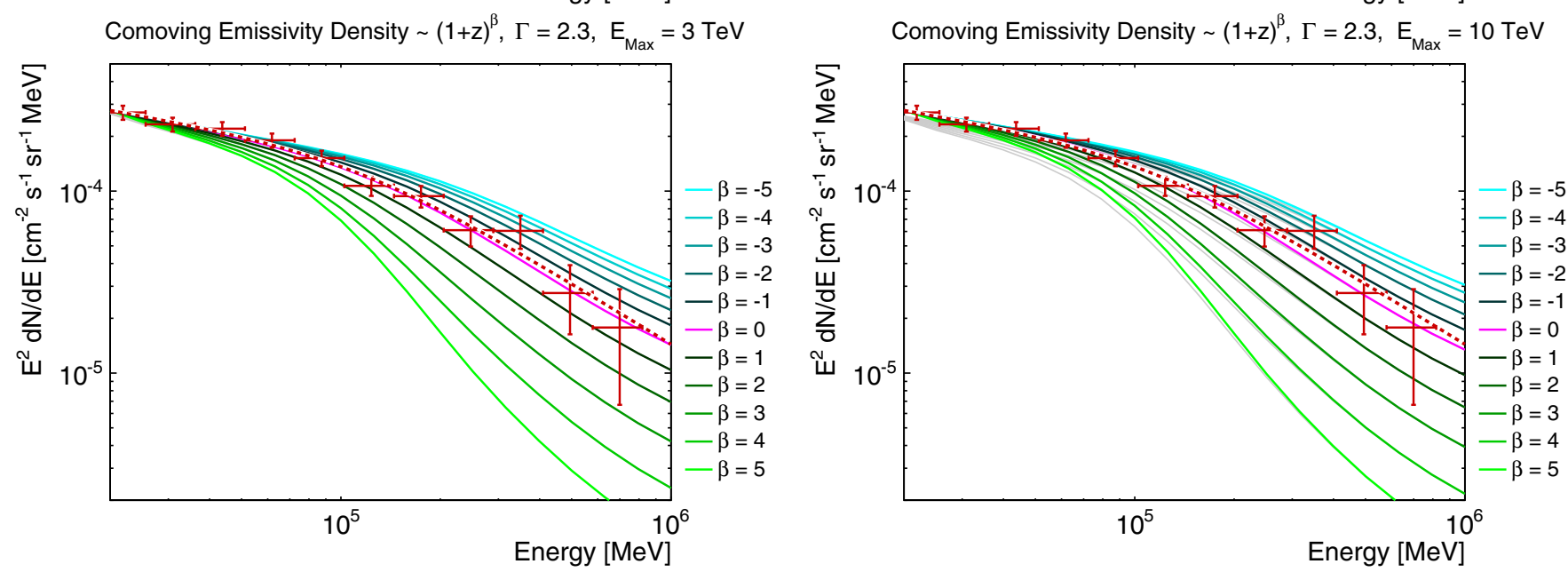

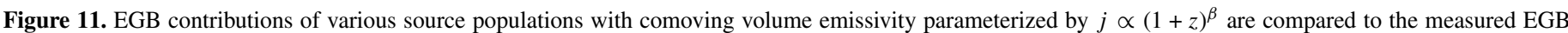

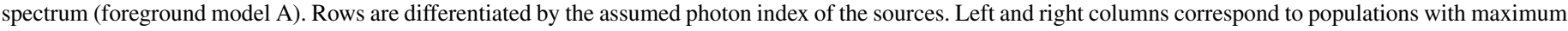

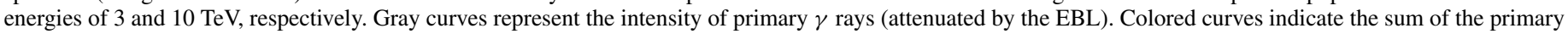
and cascade components.

constraining the abundance of sources just below the LAT detection threshold, and similar techniques may be usefully applied to LAT data in the energy range $>100 \mathrm{GeV}$.

In the interpretation that follows, we use the formalism outlined by Murase et al. (2007) and Inoue \& Ioka (2012) to calculate both the EBL-attenuated primary signal and the electromagnetic cascade emission that would arise from a variety of generic cosmologically evolving source populations. We adopt the UV/optical/IR EBL model of Franceschini et al. (2008) based on observed galaxy counts, which is found to be consistent with spectral analyses of individually detected $\gamma$-ray sources (e.g., Ackermann et al. 2012f). The populations are modeled as a collection of sources sharing the same intrinsic simple PL spectral form with a common photon index, $\gamma$, and 

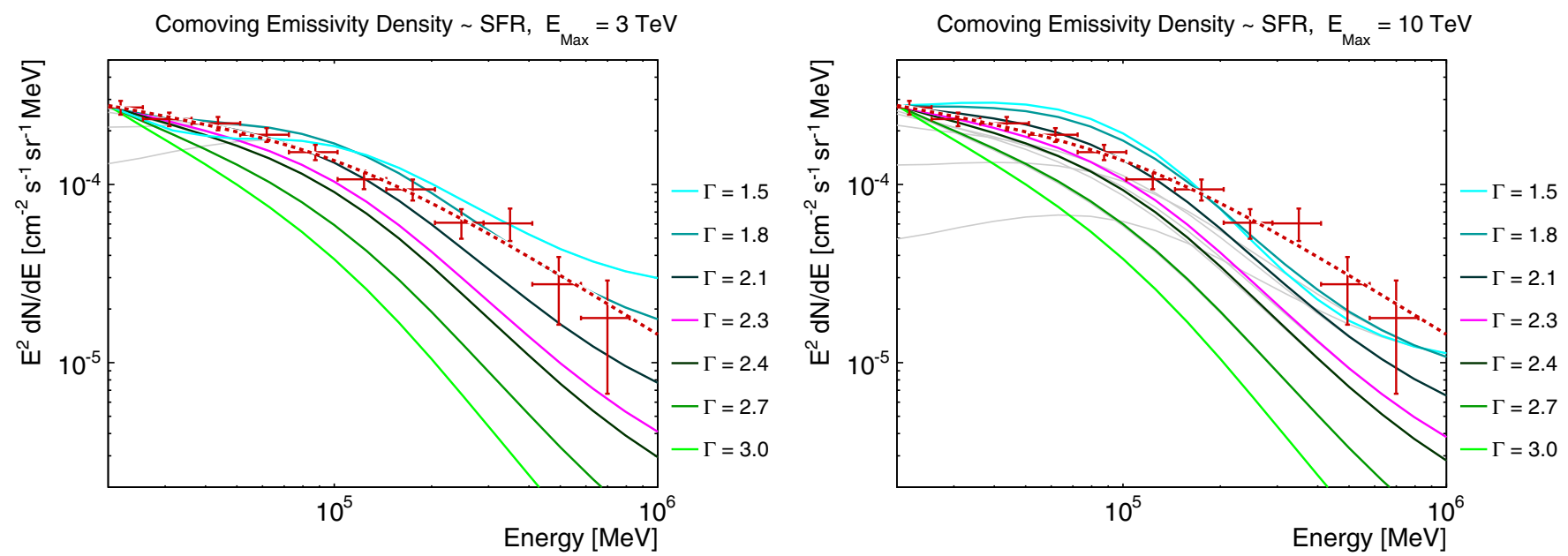

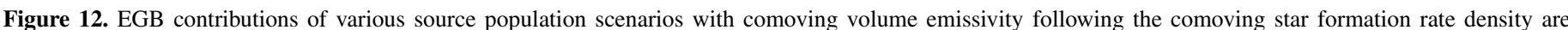

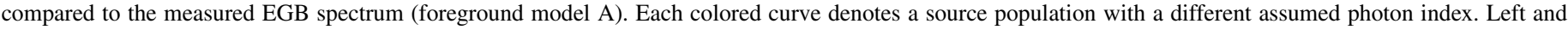

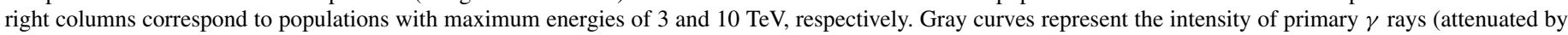
the EBL). Colored curves indicate the sum of the primary and cascade components.

maximum energy, $E_{\max }$ :

$$
d N / d E=\left\{\begin{array}{ll}
N_{0}\left(E / E_{0}\right)^{-\gamma}, & E \leqslant E_{\max } \\
0, & E>E_{\max }
\end{array},\right.
$$

where $E$ is the energy of photons emitted at the source. We model the evolving comoving volume emissivity (photons $\mathrm{s}^{-1} \mathrm{~cm}^{-3}$ ) of source populations without distinguishing between luminosity or density evolution of the sources. The emissivity evolution either (1) is parameterized by $j \propto(1+z)^{\beta}$ or (2) follows the cosmic star formation rate density (Behroozi et al. 2013). We consider sources up to redshifts $z=2$ and $z=3$ in the two cases above, respectively. Our conclusions do not qualitatively change when using a lower maximum redshift of $z=1$. No attempt is made here to identify sources that could be individually resolved by the LAT versus those that blur into the unresolved background.

The expected total EGB contributions corresponding to various source population scenarios are compared to the measured EGB spectrum $>20 \mathrm{GeV}$ in Figures 11 and 12. In each case, the contribution of the sources (i.e., sum of primary and cascade components) to the total EGB has been normalized to match that of the measured total EGB intensity at $20 \mathrm{GeV}$. Figure 11 shows source populations with parameterized comoving volume emissivities, while Figure 12 shows populations whose emissivity follows the comoving star formation rate density. In scenarios with photon index $\Gamma=1.5$, the cascade component can dominate the primary component at energies $<100 \mathrm{GeV}$.

Several patterns are apparent in Figures 11 and 12. First, source populations with negative evolution, especially those with very hard PL spectra $(\Gamma<2)$ extending to multi-TeV energies, have difficulty fitting the high-energy break in the total EGB spectrum and are therefore unlikely to account for the EGB on their own. On the other hand, source populations whose emissivity evolves as the cosmic star-forming rate (corresponding to $\beta \sim 3.25$ at low redshifts, e.g., Hopkins \& Beacom 2006) also face challenges to match the shape of the total EGB spectrum alone, unless the spectral properties of those sources are finely tuned. The source populations that would most readily explain the measured total EGB spectrum from $100 \mathrm{MeV}$ to nearly $1 \mathrm{TeV}$ are those with photon indices matching that of the EGB below $100 \mathrm{GeV}$, namely, 2.3, and little or no evolution. In fact, the distribution of photon indices for individual LAT sources detected above $10 \mathrm{GeV}$ is also peaked near a value of 2.3 (Ackermann et al. 2013c). These and similar studies (e.g., Venters 2010; Inoue \& Ioka 2012; Murase et al. 2012) demonstrate that the PL shape of the total EGB spectrum with a single cutoff/break at $\sim 250 \mathrm{GeV}$ could in principle be explained by a single dominant extragalactic source population with relatively generic spectral properties and EBL attenuation. However, more sophisticated modeling efforts taking into account the specific properties of established extragalactic source classes are needed to fully understand how sources governed by such diverse physics produce a nearly featureless EGB spectrum over $\sim 4$ decades in energy.

In addition to the implications for source classes composing the EGB, a second aspect of this work is a further examination of the DGE (Section 4). In the effort to accurately subtract the DGE and thereby isolate the fainter isotropic component, we considered a wider range of models for CR injection and propagation in the ISM of the Milky Way than previously considered, e.g., in Ackermann et al. (2012g). None of the models tested here simultaneously satisfy constraints from both local CR measurements and observations of the high-latitude $\gamma$ ray sky, particularly in the case of IC emission (see Appendix A). The lack of a clearly preferred DGE model is the largest single source of systematic uncertainty when measuring the IGRB intensity in the $100 \mathrm{MeV}$ to $100 \mathrm{GeV}$ energy range with the LAT. Some of the modifications to commonly used CR injection and diffusion treatments investigated here may provide interesting future avenues of research.

Further improvements over the Pass 7 event reconstruction and classification are required to extend EGB measurements with the LAT to both lower and higher energies. An extension to energies $\sim 50 \mathrm{MeV}$ may help to constrain the radiative processes contributing to the EGB, e.g., through the identification of the (redshifted) pionic spectral feature expected from the interactions of CR nuclei in all galaxies (e.g., Stecker \& Venters 2011; Lacki et al. 2012; Chakraborty \& Fields 2013), although no indication of such a low-energy cutoff is present in the current measurement at $\sim 100 \mathrm{MeV}$. An extension to energies $\sim 1 \mathrm{TeV}$ would further clarify the spectra and evolution 
of sources that will be studied in detail with the extragalactic surveys of the High-Altitude Water Cherenkov observatory (HAWC) ${ }^{79}$ and the Cherenkov Telescope Array (CTA). ${ }^{80}$ Both of these spectral extensions to the LAT IGRB measurement may be realized with future Pass 8 analyses (Atwood et al. 2013). Additional insight regarding the sources of the EGB may come from ongoing studies of the extragalactic background of highenergy neutrinos (Aartsen et al. 2013), since the interactions of very high energy and ultra high energy CRs inevitably create fluxes of both $\gamma$-rays and neutrinos, the implications of which are discussed by, e.g., Ahlers et al. (2010), Berezinsky et al. (2011), Wang et al. (2011), Gelmini et al. (2012), and Murase et al. (2012).

The Fermi LAT Collaboration acknowledges generous ongoing support from a number of agencies and institutes that have supported both the development and the operation of the LAT, as well as scientific data analysis. These include the National Aeronautics and Space Administration and the Department of Energy in the United States; the Commissariat à l'Energie Atomique and the Centre National de la Recherche Scientifique/Institut National de Physique Nucléaire et de Physique des Particules in France; the Agenzia Spaziale Italiana and the Istituto Nazionale di Fisica Nucleare in Italy; the Ministry of Education, Culture, Sports, Science and Technology (MEXT), High Energy Accelerator Research Organization (KEK), and Japan Aerospace Exploration Agency (JAXA) in Japan; and the K. A. Wallenberg Foundation, the Swedish Research Council, and the Swedish National Space Board in Sweden. Additional support for science analysis during the operations phase is gratefully acknowledged from the Istituto Nazionale di Astrofisica in Italy and the Centre National d'Études Spatiales in France. GALPROP development is partially funded via NASA grant NNX09AC15G. Some of the results in this paper have been derived using the HEALPix (Górski et al. 2005) package.

\section{APPENDIX A}

\section{GALACTIC FOREGROUND MODELS}

This appendix summarizes the parameters used for modeling the diffuse Galactic foreground emission in the benchmark foreground models A, B, and C. Please note that a customized version of GALPROP is needed to produce the models. The output files of the corresponding GALPROP runs are provided in an electronic data repository. Links to models A, B, and C are available.

\section{A.1. Foreground Model A}

Foreground model A uses a parameterization of the distribution of pulsars in the Galaxy (Lorimer et al. 2006) as the distribution of CR sources (see Figure 13) where CR electrons and nuclei are injected into the ISM. The diffusion coefficient for their propagation in the ISM is set to $D_{x x}=7.0 \times$ $10^{-28} \mathrm{~m}^{2} \mathrm{~s}^{-1}(R / 4 \mathrm{GV})^{0.33}$, where $R$ denotes the rigidity. The CRs are reaccelerated in the ISM with a reacceleration strength (parameterized by the Alfvén velocity $v_{A}=30 \mathrm{~km} \mathrm{~s}^{-1}$ ) that is constant throughout the Galaxy. The Galactic magnetic field is modeled according to Strong et al. (2011) using a local random field strength of $7.5 \mu \mathrm{G}$. A CR halo size of $5 \mathrm{kpc}$ is chosen. All nuclei and electrons are injected with an energy

\footnotetext{
79 http://www.hawc-observatory.org/

80 http://www.cta-observatory.org/
}

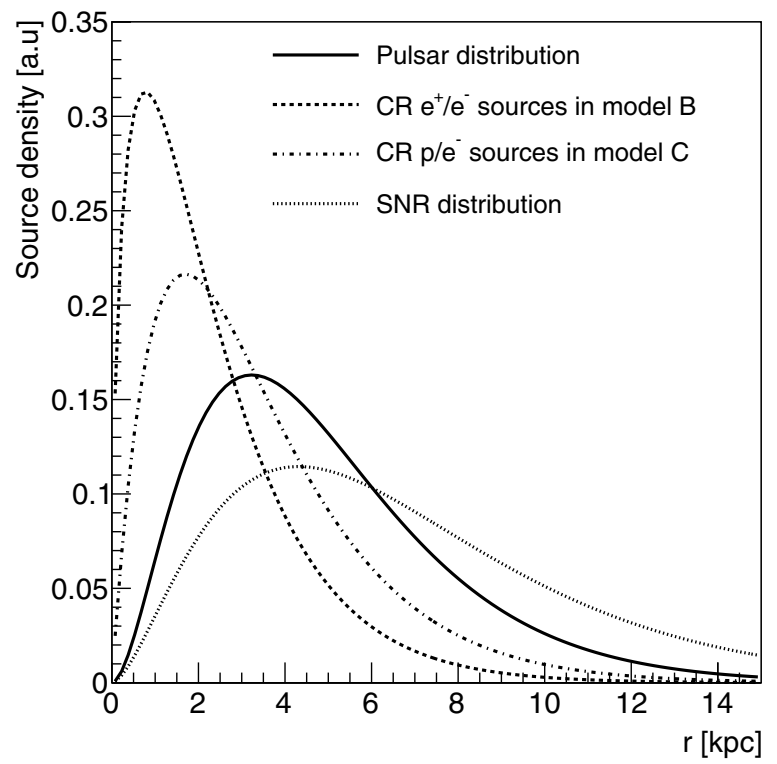

Figure 13. Parameterizations of the radial CR source distribution used in this work. The pulsar distribution is taken from Lorimer et al. (2006), the SNR distribution from Case \& Bhattacharya (1998). The curves in the figure are normalized to unit integrated source density, and the actual normalizations used in the models are derived from comparisons of predicted and measured local $\mathrm{CR}$ proton and electron intensities.

spectrum that is a BPL in rigidity. The PL index of the injection spectrum of protons is 1.9 below $9 \mathrm{GV}, 2.45$ between 9 and $240 \mathrm{GV}$, and 2.32 above $240 \mathrm{GV}$. For helium, we multiply the injection spectrum by $R^{0.1}$, and the $\mathrm{He} / \mathrm{H}$ fraction in the ISG was assumed to be 0.11 . The second break in the energy spectrum and the harder injection spectrum for helium are motivated by the results of the measurements of the proton spectrum by the PAMELA satellite (Adriani et al. 2011b) and by the CR spectrum inferred from LAT observations of Earth limb $\gamma$-rays (Ackermann et al. 2014). ${ }^{81}$ The $\gamma$-ray yield from the interactions of the CR with the ISG was calculated using the parameterization of Kamae et al. (2006).

The PL index of the injection spectrum of electrons is 1.5 below $5 \mathrm{GV}, 2.85$ between 5 and $25 \mathrm{GV}$, and 2.32 above $25 \mathrm{GV}$. As in the case of nucleons, the breaks in the spectrum are introduced to obtain a good agreement of the model with measurements of the local CR electron spectrum (Adriani et al. 2011a; Abdo et al. 2009b; Ackermann et al. 2012e). The break at $5 \mathrm{GV}$ is furthermore motivated by the shape of the Galactic synchrotron emission spectrum (Strong et al. 2011). Additionally, we assume a high-energy cutoff (implemented as a change in the injection index to 4 above $1.8 \mathrm{TeV}$ ) to remain in agreement with the HESS measurements of the electron spectrum up to several TeV (Aharonian et al. 2008).

The doubly broken injection spectra used in foreground model A improve the model/fit agreement in our maximum likelihood fit of the $\gamma$-ray data. However, there is only a negligible effect on the derived IGRB spectrum. Figure 14 shows a comparison between the expected $\gamma$-ray spectra (from foreground model A) and the spectra obtained by the maximum likelihood procedure when fitting the model templates to the $\gamma$-ray data (see also

\footnotetext{
81 We note that the AMS-02 collaboration has recently released preliminary data that do not confirm the hardening of the proton and helium spectra. A test showed that there are only negligible effects on the IGRB intensity derived in this work if we use a foreground model without a break at $240 \mathrm{GV}$ in the nucleon spectrum.
} 


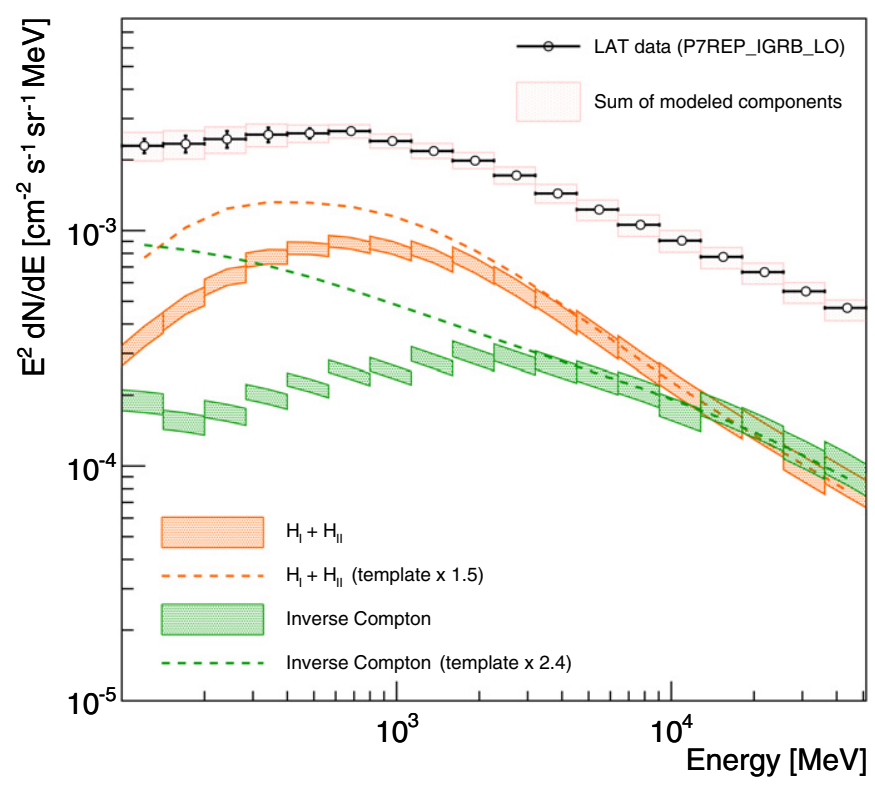

Figure 14. Fitted average intensity of the DGE in foreground model A for Galactic latitudes $|b|>20^{\circ}$. Contributions from CR interactions with the ISG and contributions from IC are shown separately. The normalizations of the two components are fitted individually in each energy bin. The GALPROP model spectrum that enters the fit is shown as dashed lines. The dashed lines are renormalized by the factors indicated in the legend.

Section 3). For the purpose of this comparison, we extend the upper bound of the energy range of the low-energy fit from 13 to $51 \mathrm{GeV}$. The $\gamma$-rays arising from CR interactions with ISG and originating from the IC process are shown separately. The input model spectra are renormalized in the figure to allow for a better comparison of the predicted and the fitted spectral shapes. The renormalization factors are determined in the energy band between 6.4 and $51 \mathrm{GeV}$ (see Section 3). The numerical values of the renormalization factors for the $\mathrm{H}$ I $+\mathrm{H}$ II and IC templates of our benchmark models are displayed in Figures 14, 16, and 18.

The predicted and observed spectra of $\gamma$-rays from CR interactions with ISG agree well at energies above a few $\mathrm{GeV}$, besides a moderate renormalization factor. A harder spectrum is seen at energies below a few $\mathrm{GeV}$ in the fit compared to the model. In this energy range, the local interstellar spectrum of CRs is difficult to measure owing to the effects of solar modulation.

The IC emission model overpredicts the fitted IC emission at low energies by a factor of up to $\sim 4$, while at high energies it underpredicts the emission by a factor of 2.4. Again, the spectral shapes of the model and the fit to LAT data show good agreement at energies above a few $\mathrm{GeV}$, so the (rescaled) model predictions from foreground model A can be used for the highenergy analysis, where the foreground model is fixed.

\section{A.2. Foreground Model B}

A significantly better agreement between the predicted and fitted IC emission is found for foreground model B, which includes an additional population of electron-only sources located near the Galactic center. In this model, the bulk of the IC emission arises from the electrons injected near the Galactic center, and the sources of CR nuclei do not produce a significant fraction of the local CR electron flux. The spatial distribution that is used for this additional electron-only source population is shown in Figure 13. We further assume that the Galactic center

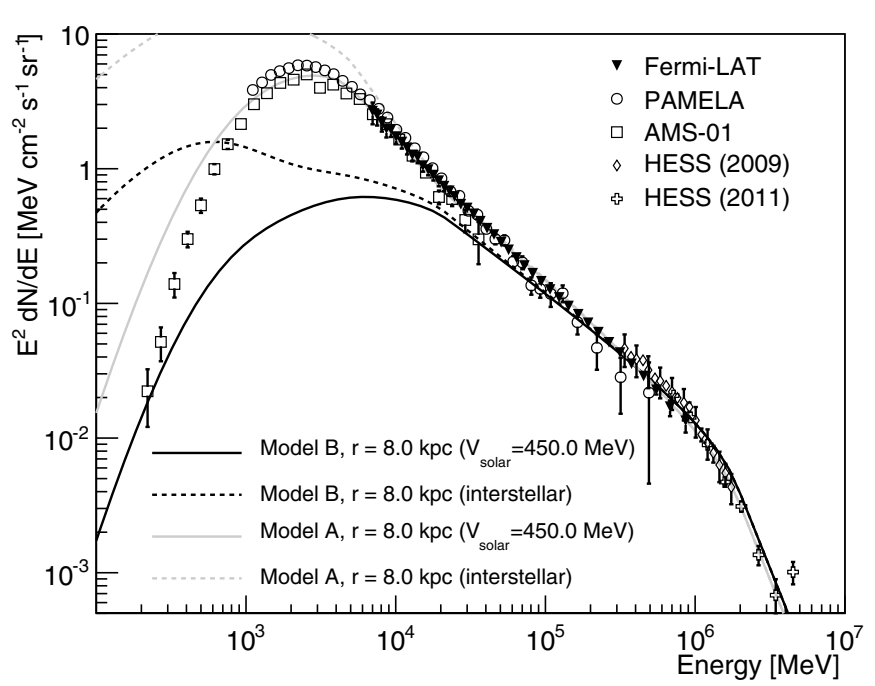

Figure 15. Predicted local CR electron spectrum of foreground models A and $\mathrm{B}$ in comparison to measurements of the spectrum. LAT data are taken from Ackermann et al. (2010), PAMELA data from Adriani et al. (2011a), AMS-01 data from Aguilar et al. (2002), and HESS data from Aharonian et al. (2009) and Egberts et al. (2011).

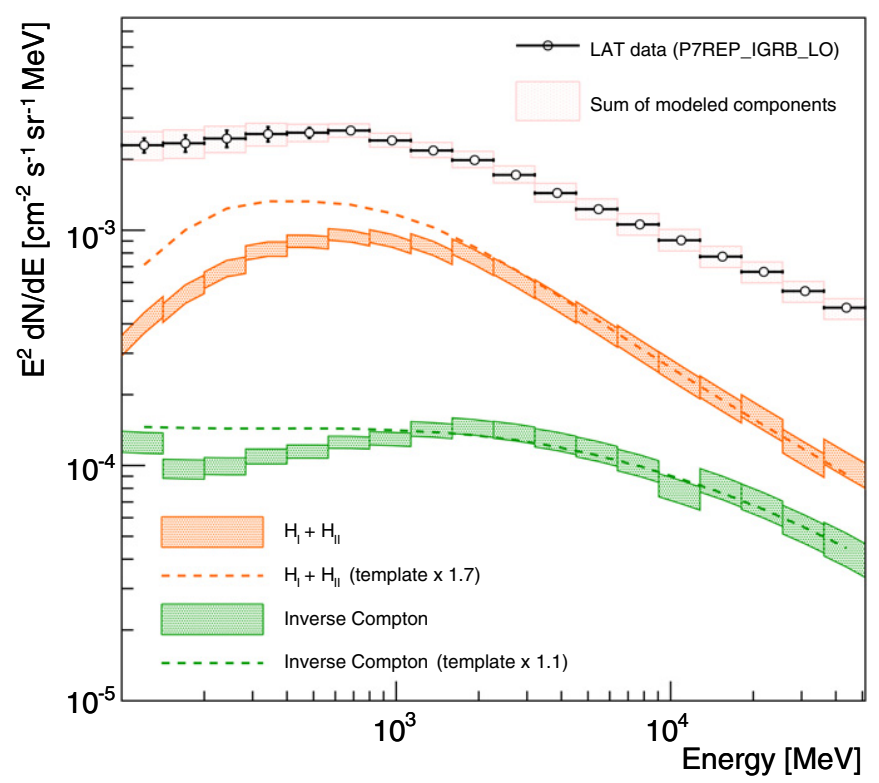

Figure 16. Fitted average intensity of the DGE in foreground model B. See Figure 14 for a description.

sources inject electrons following a PL spectrum with index 1 below $20 \mathrm{GV}$ and 2.05 above $20 \mathrm{GV}$. We also assume the same high-energy cutoff as in foreground model A to maintain agreement with HESS measurements (Aharonian et al. 2008). The additional electron source population must be located close to the Galactic center in order to produce bright enough IC emission to match $\gamma$-ray observations without overpredicting the local CR electron spectrum at high energies (assuming that the model diffusion parameters are unchanged).

Figure 15 shows the electron spectrum produced by model B in comparison to measurements, as well as the electron spectrum predicted by model A. There is a clear deficit of electrons below $20 \mathrm{GV}$ in this model. However, these electrons could be easily supplied by a local source or source population of very soft electrons without a large impact on the total amount of IC 

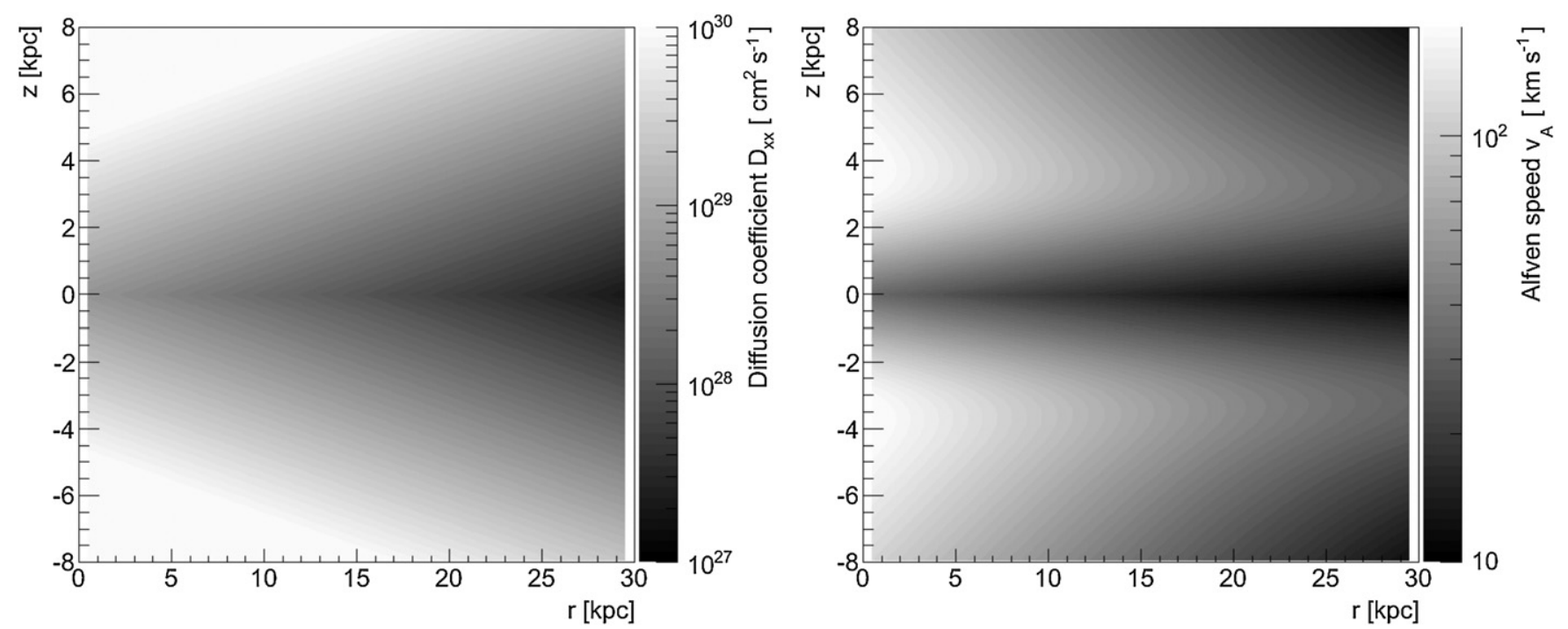

Figure 17. Diffusion coefficient (left) and Alfvén velocity (right) used in foreground model C. Both are functions of distance from the Galactic center $(r)$ and height above the Galactic plane $(z)$.

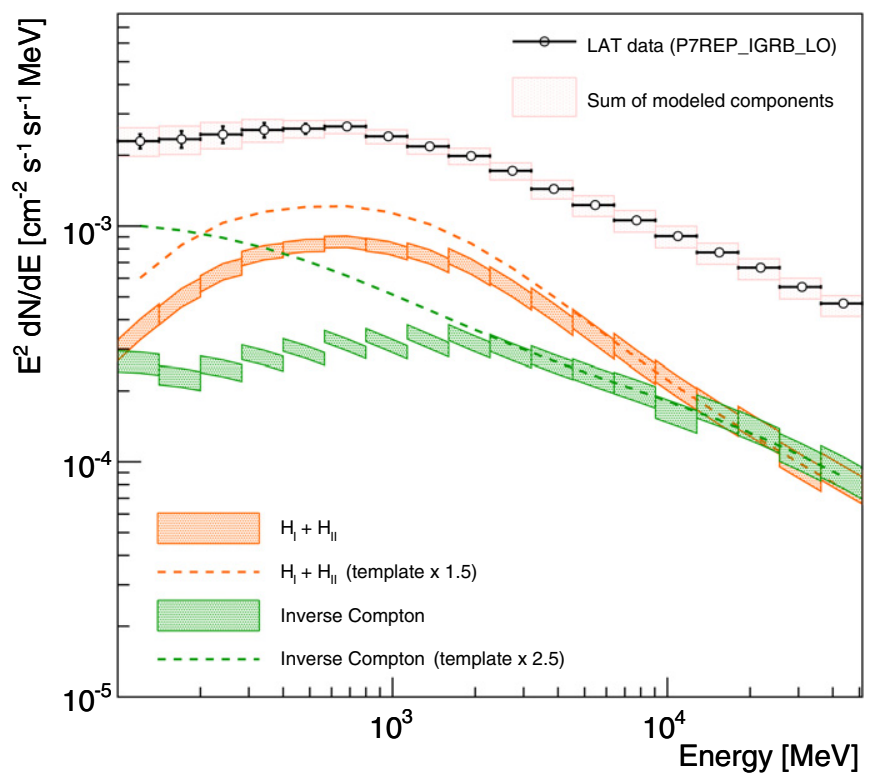

Figure 18. Fitted average intensity of the DGE in foreground model C. See Figure 14 for a description.

emission. Natural candidates would be very old SNRs in the solar neighborhood, e.g., Loop I, where we see high-energy $\gamma$ ray emission from the shell in LAT data. It is, however, beyond the scope of this paper to speculate about and address the nature of such local soft electron sources. We ignore this potential local contribution for the IGRB analysis.

The same propagation and injection parameters for nuclei are used in foreground model B as in model A. Modeled and fitted spectra are compared in Figure 16. The renormalization factor for $\gamma$-rays from the interactions of CRs with ISG increases from 1.5 in foreground model A to 1.7 in foreground model B. This is a large change, and further investigations should be undertaken to understand whether the model B value is still within the bounds of our current uncertainties concerning the total column density of the high-latitude gas, the $\gamma$-ray emissivity of the ISG, and the gradient in the CR spectrum. For the purpose of this IGRB analysis, we accept the renormalized spectrum as a valid fit of the Galactic foreground. It can be further seen from Figure 16 that the IC spectrum is now well described by the model both in normalization and in shape, besides a small discrepancy at the lowest energies.

\section{A.3. Foreground Model C}

Foreground model $\mathrm{C}$ represents a class of models in which the CR diffusion and reacceleration vary significantly throughout the Galaxy. Diffusion and reacceleration are parameterized within the transport equation implemented in GALPROP via the spatial diffusion coefficient $D_{x x}$ and the Alfvén velocity $v_{A}$. We use a simple model to vary the diffusion coefficient and Alfvén velocity by connecting their values to the strength of the regular and random Galactic magnetic fields. Following the approximation of Strong et al. (2007), the diffusion coefficient is set to

$$
\begin{aligned}
D_{x x}(R, r, z)= & D_{x x}^{0}\left(\frac{\delta B(r, z)}{\delta B_{\odot}} \times \frac{B_{\odot}}{B(r, z)}\right)^{-2} \\
& \times\left(\frac{R}{R_{0}} \times \frac{B_{\odot}}{B(r, z)}\right)^{0.33},
\end{aligned}
$$

where $B$ is the strength of the regular field and $\delta B$ corresponds to the strength of the random field at radius $r$ from the Galactic center and height $z$ above the Galactic plane. $B_{\odot}$ and $\delta B_{\odot}$ denote their values at the position of the solar system. $R_{0}=4 \mathrm{GV}$ is the reference rigidity, and $D_{x x}^{0}$ is the local diffusion coefficient at reference rigidity. The diffusion coefficient is constrained to $D_{x x}\left(R_{0}, r, z\right) \leqslant 10^{30} \mathrm{~cm}^{2} \mathrm{~s}^{-1}$ at reference rigidity. This constraint ensures that the mean free path of the CRs stays below the kiloparsec scale for particles up to tens of TV in rigidity. ${ }^{82}$

We parameterize the Alfvén velocity as $v_{A} \propto B_{\text {tot }} / \sqrt{\rho}$, where $B_{\text {tot }}$ is the total magnetic field strength and $\rho$ the density of ions

\footnotetext{
82 We tested that the specific choice of this upper bound on the diffusion coefficient is irrelevant by increasing the maximum value for $D_{x x}\left(R_{0}, r, z\right)$ by one order of magnitude. The effects on the predicted $\gamma$-ray emission were negligible.
} 

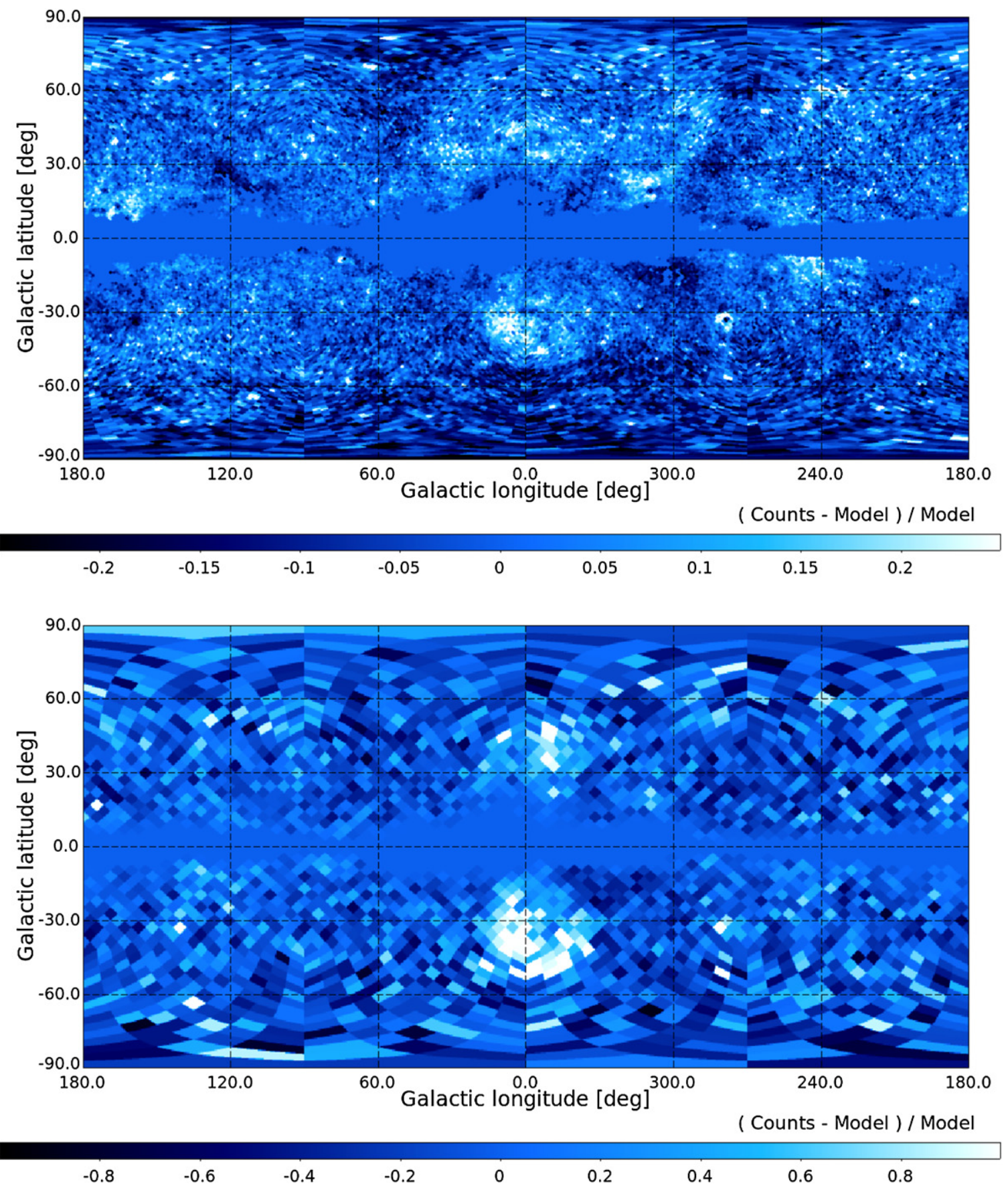

Figure 19. Residual maps for foreground model A used in this analysis. The fractional difference in counts between the actual data and the fitted model is shown in the figures. Upper: all counts above $100 \mathrm{MeV}$ are included in the map. The pixel size is $0.8 \mathrm{deg}^{2}$ (HEALPix order 6). Lower: only counts above $13 \mathrm{GeV}$ are included in the map. The pixel size has been increased to $13 \mathrm{deg}^{2}$ (HEALPix order 4) to account for the reduced count statistics at higher energies.

in the ISM. For the ion density, the same model is used as in the rest of this work (Gaensler et al. 2008). Simple models are assumed for the random and the regular magnetic field components with exponential scale heights and scale lengths. The regular magnetic field strength is assumed to be $4 \mu \mathrm{G}$ at the position of the Sun, with a scale length of $11 \mathrm{kpc}$ and a scale height of $4 \mathrm{kpc}$. The random field strength is assumed to be $4 \mu \mathrm{G}$, constant in the Galactic plane with a scale height of $2 \mathrm{kpc}$. The scale heights are in good agreement with scale heights found from equilibrium conditions (Kalberla \& Kerp 1998). The field strengths are in qualitative agreement with recent studies of the Galactic synchrotron emission in Orlando
\& Strong (2013), which take radio polarization into account. The extent of the CR halo for this model is set to $8 \mathrm{kpc}$; we note that constraints on the halo size found in earlier studies based on the ${ }^{10} \mathrm{Be} /{ }^{9} \mathrm{Be}$ ratio apply only to models with a static diffusion coefficient. Figure 17 shows the diffusion coefficient and the Alfvén velocity as a function of the galactocentric radius $r$ and the height above the Galactic plane $z$.

A customized version of the GALPROP code (see above) is used that allows the modeling of propagation scenarios in which the spatial and momentum diffusion are functions of radial distance from the Galactic center and height above the Galactic plane. As for foreground models A and B, GALPROP 


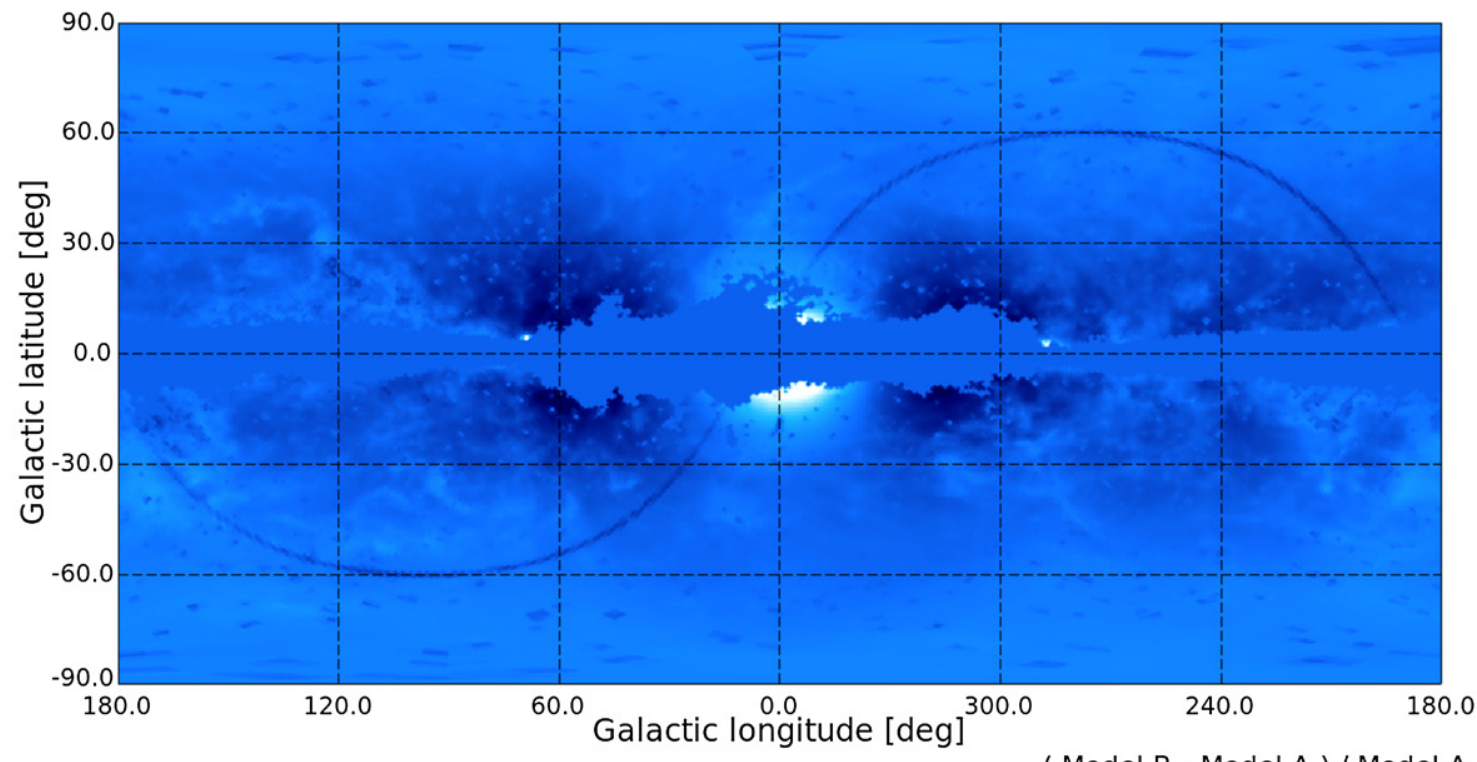

( Model B - Model A ) / Model A
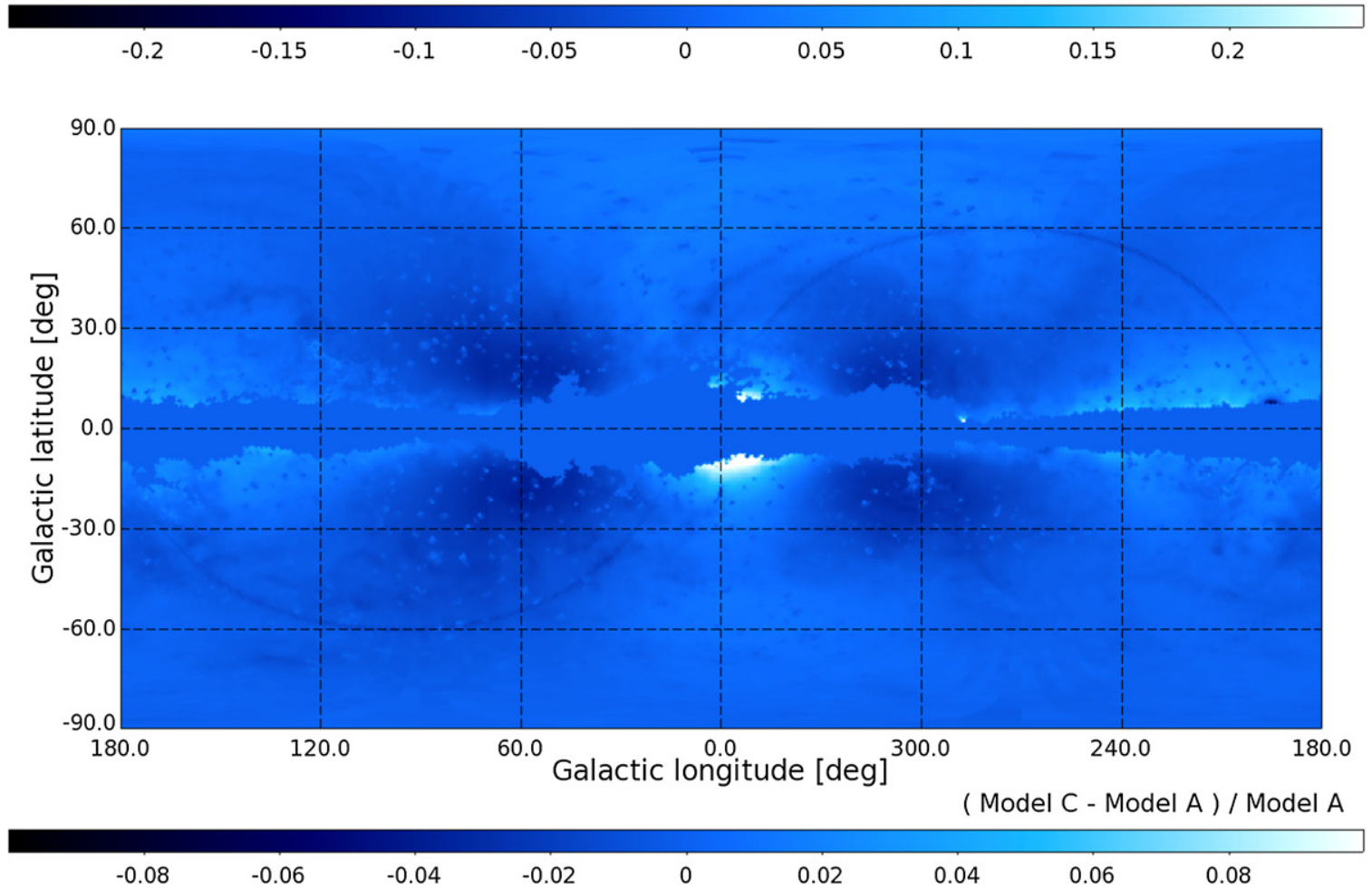

Figure 20. Differences in foreground models A, B, and C. The fractional differences in the number of predicted counts above $3 \mathrm{GeV}$ between the models using the alternative Galactic foregrounds B and C and the model using Galactic foreground A are displayed in the maps. The pixel size is $0.8 \mathrm{deg}^{2}$ (HEALPix order 6). Upper: fractional difference for model B. Lower: fractional difference for model C.

is used in its $2 \mathrm{D}$ mode that solves the transport equation on a $2 \mathrm{D}$ spatial grid in galactocentric radius and height $(r, z)$ around the Galactic center. The CR source distribution assumed in model $\mathrm{C}$ is more peaked toward the Galactic center than the pulsar distribution in model A (see Figure 13). The high-energy injection spectra for $\mathrm{CR}$ electrons and protons are the same as for model A, PLs in rigidity with an index of 2.32. However, an injection spectrum with an index of 1.9 below $13 \mathrm{GV}$ and 2.40 between 13 and $240 \mathrm{GV}$ is used for the CR protons in model C, slightly different from the spectrum used in model A. For the CR electrons the injection spectral index is 1.5 below
4.5 GV and 2.70 between 4.5 and $25 \mathrm{GV}$. These modifications in the injection spectrum improve the agreement of the local CR spectra predicted by model $\mathrm{C}$ with measurements.

Modeled and fitted $\gamma$-ray spectra are compared in Figure 18. The renormalization factor for $\gamma$-rays from the interactions of CRs with the ISG is 1.5 as for model A. The total intensity of the IC emission is similarly underpredicted by a factor of 2.5 at energies above a few $\mathrm{GeV}$, while it is overpredicted at low energies. However, an interesting aspect of this model is that it predicts a flatter CR gradient than models A and B and therefore predicts a higher $\gamma$-ray emissivity in the outer Galaxy. 
It was found in two other studies (Abdo et al. 2010a; Ackermann et al. 2011b) that the emissivity derived from LAT observations is indeed higher in the outer Galaxy than predicted by diffuse emission models of class $\mathrm{A}$. We do not discuss the $\mathrm{CR}$ gradient of model $\mathrm{C}$ further here as it is not relevant for the IGRB analysis.

\section{APPENDIX B}

\section{RESIDUAL MAPS}

Figure 19 shows residual maps of the relative deviations in the number of expected and observed counts when using foreground model A for the DGE. The first map shows the residual for all counts above $100 \mathrm{MeV}$, while the second map shows the residual for counts above $13 \mathrm{GeV}$. Multiple structures are visible in the former map, while the latter is dominated by the Fermi bubbles.

Figure 20 visualizes the difference in the predicted $\gamma$-ray emission when using Galactic foreground models B or C instead of model A. These differences are more prominent at energies above a few $\mathrm{GeV}$, where the IC emission contributes a larger fraction of the total $\gamma$-ray emission than at a few hundreds of $\mathrm{MeV}$. Therefore, the relative deviation in predicted counts above $3 \mathrm{GeV}$ is shown in the maps when using foreground models $\mathrm{B}$ and $\mathrm{C}$ in the fit with respect to using foreground model A.

For foreground model $\mathrm{B}$, a higher $\gamma$-ray intensity is predicted close to the Galactic center region arising from the IC emission of electrons that originate from the additional source population we introduced in this model. For foreground model $\mathrm{C}$, regions with higher intensity can be observed toward the outer Galaxy, reflecting the more efficient transport of CRs into the outer Galaxy by the modified propagation scheme used in model C.

\section{APPENDIX C}

\section{CRITERIA FOR MASKING REGIONS IN THE LIKELIHOOD FIT}

Approximately $90 \%$ of the ISG is atomic ( $\mathrm{HI})$, ionized (HII), or molecular hydrogen gas $\left(\mathrm{H}_{2}\right)$. The remainder is mostly helium and is generally assumed to be mixed uniformly with the hydrogen. The column density and distribution of atomic gas in the Galaxy can be estimated from surveys of the $21 \mathrm{~cm}$ hyperfine-structure transition line of the hydrogen atom (Kalberla et al. 2005). The distribution of molecular hydrogen gas can be inferred indirectly from surveys of the $2.6 \mathrm{~mm} J(1 \rightarrow 0)$ transition of the CO molecule by assuming a proportionality (usually called the $X_{\mathrm{CO}}$ factor) between the intensity of this line integrated over frequency $\left(W_{\mathrm{CO}}\right)$ and the column density of $\mathrm{H}_{2}$ gas (Dame et al. 2001). In both cases, the velocity component of the gas parallel to the line of sight is measured via the Doppler shift of the transition line. When combined with a model of the Galaxy rotation curve, the observed velocity can be converted into a measurement of galactocentric radius, allowing for a determination of the gas density distribution along the line of sight. In this work, we use the gas distribution model of Ackermann et al. (2012g), where the total gas column density is distributed in 17 galactocentric rings spanning the radial range from 0 to $50 \mathrm{kpc}$. Owing to the small scale heights of the gas (a few tens of parsecs for $\mathrm{H}_{2}$ and a few hundred parsecs for $\mathrm{HI}$ ), most of the gas outside of our local Galactic neighborhood will appear concentrated around the Galactic plane. Even in our local neighborhood, most of the $\mathrm{H}_{2}$ gas is concentrated in isolated clouds at low Galactic latitudes.
We use this fact to exclude regions of the sky from our likelihood fit that have a significant column density of $\mathrm{H}_{2}$ gas $\left(W_{\mathrm{CO}}>2.5 \mathrm{~K} \mathrm{~km} \mathrm{~s}^{-1}\right)$ along the respective line of sight, as well as lines of sight with a significant column density of $\mathrm{H} \mathrm{I}$ gas $\left(N_{\mathrm{H} \text { I }}>5 \times 10^{20} \mathrm{~cm}^{-2}\right)$ located beyond our local solar neighborhood (8 kpc $<r<10 \mathrm{kpc}$ ) according to the gas distribution model in Ackermann et al. (2012g). Independent of the gas column densities found, Galactic latitudes $|b|<5^{\circ}$ are also excluded. The exclusion of such regions simplifies the likelihood analysis considerably. Specifically, models of the DGE in the remaining mostly high-latitude parts of the sky do not depend on assumptions about the $\mathrm{CO}$-to- $\mathrm{H}_{2}$ conversion factor $X_{\mathrm{CO}}$, or on how accurately we model variations of the CR density throughout the Galaxy, leading to variations of the $\mathrm{H}$ I emissivity for gas at larger distances. The excluded regions cover a total of $17 \%$ of the sky.

\section{REFERENCES}

Aartsen, M. G., Abbasi, R., Abdou, Y., et al. 2013, Sci, 342, 1242856 Abdo, A. A., Ackermann, M., Ajello, M., et al. 2009a, PhRvD, 80, 122004 Abdo, A. A., Ackermann, M., Ajello, M., et al. 2009b, PhRvL, 102, 181101 Abdo, A. A., Ackermann, M., Ajello, M., et al. 2009c, APh, 32, 193 Abdo, A. A., Ackermann, M., Ajello, M., et al. 2010a, ApJ, 710, 133 Abdo, A. A., Ackermann, M., Ajello, M., et al. 2010b, PhRvL, 104, 101101 Abdo, A. A., Ackermann, M., Ajello, M., et al. 2010c, ApJ, 720, 435 Abdo, A. A., Ackermann, M., Ajello, M., et al. 2011, ApJ, 734, 116 Abdo, A. A., Ackermann, M., Ajello, M., et al. 2012, ApJ, 758, 140 Abdo, A. A., Ajello, M., Allafort, A., et al. 2013, ApJS, 208, 17 Ackermann, M., Ajello, M., Albert, A., et al. 2012a, PhRvD, 85, 083007 Ackermann, M., Ajello, M., Albert, A., et al. 2012b, ApJS, 203, 4 Ackermann, M., Ajello, M., Albert, A., et al. 2014, PhRvL, 112, 151103 Ackermann, M., Ajello, M., Allafort, A., et al. 2011a, ApJ, 743, 171 Ackermann, M., Ajello, M., Allafort, A., et al. 2012c, ApJ, 755, 164 Ackermann, M., Ajello, M., Allafort, A., et al. 2012d, APh, 35, 346 Ackermann, M., Ajello, M., Allafort, A., et al. 2012e, PhRvL, 108, 011103 Ackermann, M., Ajello, M., Allafort, A., et al. 2012f, Sci, 338, 1190 Ackermann, M., Ajello, M., Allafort, A., et al. 2013a, ApJ, 765, 54 Ackermann, M., Ajello, M., Allafort, A., et al. 2013b, ApJ, 778, 82 Ackermann, M., Ajello, M., Allafort, A., et al. 2013c, ApJS, 209, 34 Ackermann, M., Ajello, M., Atwood, W. B., et al. 2010, PhRvD, 82, 092004 Ackermann, M., Ajello, M., Atwood, W. B., et al. 2012g, ApJ, 750, 3 Ackermann, M., Ajello, M., Baldini, L., et al. 2011b, ApJ, 726, 81

Ackermann, M., Jóhannesson, G., Digel, S. W., et al. 2009, AIP Conf. Proc. 1085, High Energy Gamma-ray Astronomy, ed. F. A. Aharonian, W. Hofmann, \& F. M. Rieger (Melville, NY: AIP), 763

Adriani, O., Barbarino, G. C., Bazilevskaya, G. A., et al. 2011a, PhRvL, 106,201101

Adriani, O., Barbarino, G. C., Bazilevskaya, G. A., et al. 2011b, Sci, 332, 69

Aguilar, M., Alcaraz, J., Allaby, J., et al. 2002, PhR, 366, 331

Aharonian, F., Akhperjanian, A. G., Anton, G., et al. 2009, A\&A, 508, 561

Aharonian, F., Akhperjanian, A. G., Barres de Almeida, U., et al. 2008, PhRvL, 101,261104

Ahlers, M., Anchordoqui, L. A., Gonzalez-Garcia, M. C., Halzen, F., \& Sarkar, S. 2010, APh, 34, 106

Ajello, M., Greiner, J., Sato, G., et al. 2008, ApJ, 689, 666

Ajello, M., Shaw, M. S., Romani, R. W., et al. 2012, ApJ, 751, 108

Ajello, M., Romani, R. W., Gasparrini, D., et al. 2014, ApJ, 780, 73

Atwood, W., Albert, A., Baldini, L., et al. 2013, arXiv:1303.3514

Atwood, W. B., Abdo, A. A., Ackermann, M., et al. 2009, ApJ, 697, 1071

Bechtol, K. 2012, PhD thesis, Stanford Univ.

Behroozi, P. S., Wechsler, R. H., \& Conroy, C. 2013, ApJ, 770, 57

Berezinskii, V. S., \& Smirnov, A. I. 1975, Ap\&SS, 32, 461

Berezinsky, V., Gazizov, A., Kachelrieß, M., \& Ostapchenko, S. 2011, PhLB, 695,13

Bergström, L., Edsjö, J., \& Ullio, P. 2001, PhRvL, 87, 251301

Bregeon, J., Charles, E., \& Wood, M. 2013, arXiv:1304.5456

Casandjian, J.-M., \& Grenier, I. 2009, arXiv:0912.3478

Case, G. L., \& Bhattacharya, D. 1998, ApJ, 504, 761

Chakraborty, N., \& Fields, B. D. 2013, ApJ, 773, 104

Churazov, E., Sunyaev, R., Revnivtsev, M., et al. 2007, A\&A, 467, 529

Clark, G. W., Garmire, G. P., \& Kraushaar, W. L. 1968, ApJL, 153, L203

Colafrancesco, S., \& Blasi, P. 1998, APh, 9, 227 
Coppi, P. S., \& Aharonian, F. A. 1997, ApJL, 487, L9

Dame, T. M., Hartmann, D., \& Thaddeus, P. 2001, ApJ, 547, 792

de Palma, F., Brandt, T. J., Johannesson, G., \& Tibaldo, L. 2013, arXiv: 1304.1395

Dermer, C. D. 2007, AIP Conf. Proc. 921, The First Glast Symposium, ed. S. Ritz, P. Michelson, \& C. Meegan (Melville, NY: AIP), 122

Di Mauro, M., Calore, F., Donato, F., Ajello, M., \& Latronico, L. 2014, ApJ, 780,161

Dobler, G., Finkbeiner, D. P., Cholis, I., Slatyer, T., \& Weiner, N. 2010, ApJ, 717,825

Egberts, K., et al. 2011, NIMPA, 630, 36

Faucher-Giguère, C.-A., \& Loeb, A. 2010, JCAP, 1, 5

Feldmann, R., Hooper, D., \& Gnedin, N. Y. 2013, ApJ, 763, 21

Fermi LAT Collaboration. 2014, ApJ, 793, 64

Fichtel, C. E., Hartman, R. C., Kniffen, D. A., et al. 1975, ApJ, 198, 163

Fichtel, C. E., Simpson, G. A., \& Thompson, D. J. 1978, ApJ, 222, 833

Fields, B. D., Pavlidou, V., \& Prodanović, T. 2010, ApJL, 722, L199

Franceschini, A., Rodighiero, G., \& Vaccari, M. 2008, A\&A, 487, 837

Fukada, Y., Hayakawa, S., Kasahara, I., et al. 1975, Natur, 254, 398

Gaensler, B. M., Madsen, G. J., Chatterjee, S., \& Mao, S. A. 2008, PASA, 25,184

Gelmini, G. B., Kalashev, O., \& Semikoz, D. V. 2012, JCAP, 1, 44

Gendreau, K. C., Mushotzky, R., Fabian, A. C., et al. 1995, PASJ, 47, L5

Górski, K. M., Hivon, E., Banday, A. J., et al. 2005, ApJ, 622, 759

Grenier, I. A., Casandjian, J.-M., \& Terrier, R. 2005, Sci, 307, 1292

Gruber, D. E., Matteson, J. L., Peterson, L. E., \& Jung, G. V. 1999, ApJ, 520,124

Haslam, C. G. T., Salter, C. J., Stoffel, H., \& Wilson, W. E. 1982, A\&AS, 47, 1

Healey, S. E., Romani, R. W., Taylor, G. B., et al. 2007, ApJS, 171, 61

Hopkins, A. M., \& Beacom, J. F. 2006, ApJ, 651, 142

Inoue, Y. 2011, ApJ, 733, 66

Inoue, Y., \& Ioka, K. 2012, PhRvD, 86, 023003

James, F., \& Roos, M. 1975, CoPhC, 10, 343

Johannesson, G., \& Orlando, E. 2013, arXiv:1307.0197

Kalberla, P. M. W., Burton, W. B., Hartmann, D., et al. 2005, A\&A, 440, 775

Kalberla, P. M. W., \& Kerp, J. 1998, A\&A, 339, 745

Kamae, T., Karlsson, N., Mizuno, T., Abe, T., \& Koi, T. 2006, ApJ, 647, 692

Keshet, U., Waxman, E., \& Loeb, A. 2004, JCAP, 4, 6

Kinzer, R. L., Jung, G. V., Gruber, D. E., Matteson, J. L., \& Peterson, L. E. 1997, ApJ, 475, 361

Kraushaar, W. L., Clark, G. W., Garmire, G. P., et al. 1972, ApJ, 177, 341
Kulkarni, S. R., \& Heiles, C. 1988, in Galactic and Extragalactic Radio Astronomy (2nd ed.; Berlin: Springer-Verlag), 95

Lacki, B. C., Horiuchi, S., \& Beacom, J. F. 2012, ApJ, 786, 40

Loeb, A., \& Waxman, E. 2000, Natur, 405, 156

Lorimer, D. R., Faulkner, A. J., Lyne, A. G., et al. 2006, MNRAS, 372, 777

Makiya, R., Totani, T., \& Kobayashi, M. A. R. 2011, ApJ, 728, 158

Malyshev, D., \& Hogg, D. W. 2011, ApJ, 738, 181

Moskalenko, I. V., \& Porter, T. A. 2009, ApJL, 692, L54

Moskalenko, I. V., Porter, T. A., \& Digel, S. W. 2006, ApJL, 652, L65

Murase, K., Asano, K., \& Nagataki, S. 2007, ApJ, 671, 1886

Murase, K., Beacom, J. F., \& Takami, H. 2012, JCAP, 8, 30

Nolan, P. L., Abdo, A. A., Ackermann, M., et al. 2012, ApJS, 199, 31

Orlando, E., \& Strong, A. 2013, MNRAS, 436, 2127

Orlando, E., \& Strong, A. W. 2007, Ap\&SS, 309, 359

Orlando, E., \& Strong, A. W. 2008, A\&A, 480, 847

Pavlidou, V., \& Fields, B. D. 2002, ApJL, 575, L5

Porter, T. A., Moskalenko, I. V., Strong, A. W., Orlando, E., \& Bouchet, L. 2008, ApJ, 682, 400

Revnivtsev, M., Gilfanov, M., Sunyaev, R., Jahoda, K., \& Markwardt, C. 2003, A\&A, 411, 329

Schlegel, D. J., Finkbeiner, D. P., \& Davis, M. 1998, ApJ, 500, 525

Singal, J., Petrosian, V., \& Ajello, M. 2012, ApJ, 753, 45

Sreekumar, P., Bertsch, D. L., Dingus, B. L., et al. 1998, ApJ, 494, 523

Stecker, F. W., \& Venters, T. M. 2011, ApJ, 736, 40

Strong, A. W., Moskalenko, I. V., \& Ptuskin, V. S. 2007, ARNPS, 57, 285

Strong, A. W., Moskalenko, I. V., \& Reimer, O. 2000, ApJ, 537, 763

Strong, A. W., Moskalenko, I. V., \& Reimer, O. 2004, ApJ, 613, 956

Strong, A. W., Orlando, E., \& Jaffe, T. R. 2011, A\&A, 534, A54

Su, M., Slatyer, T. R., \& Finkbeiner, D. P. 2010, ApJ, 724, 1044

Taylor, J. E., \& Silk, J. 2003, MNRAS, 339, 505

Thompson, T. A., Quataert, E., \& Waxman, E. 2007, ApJ, 654, 219

Ullio, P., Bergström, L., Edsjö, J., \& Lacey, C. 2002, PhRvD, 66, 123502

Venters, T. M. 2010, ApJ, 710, 1530

Vladimirov, A. E., Digel, S. W., Jóhannesson, G., et al. 2011, CoPhC, 182, 1156

Wang, X.-Y., Liu, R.-Y., \& Aharonian, F. 2011, ApJ, 736, 112

Watanabe, K., Hartmann, D. H., Leising, M. D., et al. 1997, in AIP Conf. Ser. 410, Proceedings of the Fourth Compton Symposium, ed. C. D. Dermer, M. S. Strickman, \& J. D. Kurfess, 1223

Weidenspointner, G., Varendorff, M., Kappadath, S. C., et al. 2000, in AIP Conf. Ser. 510, The Fifth Compton Symposium, ed. M. L. McConnell \& J. M. Ryan (Melville, NY: AIP), 467

Wolleben, M. 2007, ApJ, 664, 349 